\title{
Astrometry and exoplanets in the Gaia era: a Bayesian approach to detection and parameter recovery
}

\author{
P. Ranalli ${ }^{1,2}$, D. Hobbs ${ }^{1}$, and L. Lindegren ${ }^{1}$ \\ ${ }^{1}$ Lund Observatory, Box 43, 22100 Lund, Sweden \\ e-mail: piero@astro.lu.se \\ 2 INAF - Osservatorio Astronomico di Bologna, via Gobetti 93/3, 40129 Bologna, Italy
}

Received 3 April 2017 / Accepted 25 January 2018

\begin{abstract}
The Gaia mission is expected to make a significant contribution to the knowledge of exoplanet systems, both in terms of their number and of their physical properties. We develop Bayesian methods and detection criteria for orbital fitting, and revise the detectability of exoplanets in light of the in-flight properties of Gaia. Limiting ourselves to one-planet systems as a first step of the development, we simulate Gaia data for exoplanet systems over a grid of S/N, orbital period, and eccentricity. The simulations are then fit using Markov chain Monte Carlo methods. We investigate the detection rate according to three information criteria and the $\Delta \chi^{2}$. For the $\Delta \chi^{2}$, the effective number of degrees of freedom depends on the mission length. We find that the choice of the Markov chain starting point can affect the quality of the results; we therefore consider two limit possibilities: an ideal case, and a very simple method that finds the starting point assuming circular orbits. We use 6644 and 4402 simulations to assess the fraction of false positive detections in a $5 \mathrm{yr}$ and in a $10 \mathrm{yr}$ mission, respectively; and 4968 and 4706 simulations to assess the detection rate and how the parameters are recovered. Using Jeffreys' scale of evidence, the fraction of false positives passing a strong evidence criterion is $\lesssim 0.2 \%(0.6 \%)$ when considering a $5 \mathrm{yr}(10 \mathrm{yr})$ mission and using the Akaike information criterion or the WatanabeAkaike information criterion, and $<0.02 \%(<0.06 \%)$ when using the Bayesian information criterion. We find that there is a $50 \%$ chance of detecting a planet with a minimum $S / N=2.3(1.7)$. This sets the maximum distance to which a planet is detectable to $\sim 70 \mathrm{pc}$ and $\sim 3.5 \mathrm{pc}$ for a Jupiter-mass and Neptune-mass planets, respectively, assuming a $10 \mathrm{yr}$ mission, a 4 au semi-major axis, and a $1 M_{\odot}$ star. We show the distribution of the accuracy and precision with which orbital parameters are recovered. The period is the orbital parameter that can be determined with the best accuracy, with a median relative difference between input and output periods of $4.2 \%(2.9 \%)$ assuming a $5 \mathrm{yr}(10 \mathrm{yr})$ mission. The median accuracy of the semi-major axis of the orbit can be recovered with a median relative error of $7 \%(6 \%)$. The eccentricity can also be recovered with a median absolute accuracy of $0.07(0.06)$
\end{abstract}

Key words. methods: statistical - astrometry - methods: numerical - techniques: miscellaneous - celestial mechanics planetary systems

\section{Introduction}

The detection of exoplanets with astrometric techniques is simple in principle. Although an exoplanet is usually too faint to be observed with current instruments, its existence can be inferred from the motion of its host star around the centre of mass of the star-planet system. In the past, several claims of detections have been made and later disproved starting from Jacob (1855) (see Quirrenbach 2010 for a brief historical account). The difficulty lies in the small magnitude of the host star astrometric shift, which even in the most favourable cases is $\lesssim 1$ mas (see e.g. Sect. 3).

The astrometric satellite HIPPARCOS (ESA 1997) could measure the along-scan position of a star with an error of a few mas. Several studies tried to derive orbital constraints for candidate planets suggested by radial velocity searches (Mazeh et al. 1999; Zucker \& Mazeh 2000; Gatewood et al. 2001; Han et al. 2001), but fitting orbital models to HIPPARCOS data hardly improved the astrometric fit and led instead to spurious features (Pourbaix 2001).
The Gaia mission (Gaia Collaboration 2016b) offers a $~ 30$ fold improvement in astrometric precision with respect to HIPPARCOS, and can therefore finally allow the astrometric detection of planets to become routine. The Gaia capabilities for planet detection were soon recognised (Bernstein \& Bastian 1995; Casertano et al. 1995), and explored in depth by Casertano et al. (2008, hereafter C08) and later revised by Perryman et al. (2014, hereafter P14). The number of detectable planets was estimated by P14 to be $\sim 21000$ for a $5 \mathrm{yr}$ mission, after considering a model of the Galaxy population and fitting planetary orbits with the least-squares method.

In this paper we consider the application of Bayesian methods to the problem of planetary orbit fitting. We focus on two aspects of Bayesian inference: the derivation of joint errors for all parameters of interest, and the comparison of models. The first builds on the ability of Markov chain Monte Carlo (MCMC) methods to track errors beyond the regime in which linear approximations are acceptable. The second is especially important in light of the difficulties that arise for calculating goodness of fit for non-linear models. Our discussion also includes a 
traditional metric, $\Delta \chi^{2}$, that can be used to achieve results similar to those obtained using information criteria. Bayesian methods are already in use for the Gaia data processing, and thus we expect our results to better anticipate the outcome of future Gaia data releases.

Similarly to $\mathrm{C} 08$ and at variance with P14, we focus more on the detectability as a function of the orbital characteristics than on the number of detectable planets in the solar neighbourhood. While C08 was published five years before the launch of Gaia and therefore reflects pre-launch expectations, in this paper we use the current knowledge of the satellite to reassess what fraction of planets can be detected and how well their orbital parameters can be recovered.

In this paper we focus on developing methods, and so we only consider single-planet systems. While this may be a reasonable approximation in some cases, real-world planetary systems can have more than one planet with a non-negligible $\mathrm{S} / \mathrm{N}$, in which case the results discussed here may not apply. Double-planet and more complicated systems will be considered in a follow-up paper.

The structure of this paper is as follows. In Sect. 2 we describe our adopted formalism for the description of Keplerian orbits in an astrometry setting. In Sect. 3 we consider the expected $\mathrm{S} / \mathrm{N}$ for a one-planet system. In Sect. 4 we describe our simulations. In Sect. 5 we consider Bayesian methods and detection metrics. In Sect. 6 we estimate the fraction of false positive detections. In Sect. 7 we show the detection rate. In Sect. 8 we discuss how the orbital parameters are recovered. In Sect. 9 we discuss our results, and include an assessment of the average accuracy and precision which a parameter can be recovered. Finally, in Sect. 10, we present our conclusions.

\section{Astrometry and Keplerian orbits}

\subsection{Formalism}

An exoplanet orbit can be determined in the same way as that of astrometric binary stars, where only one element of the binary system is visible. Here we summarise the method; secondary references include Binnendijk (1960), Taff (1985), Quirrenbach (2010), and Perryman (2011).

Let us consider a system made of a star and one planet. In an inertial reference frame, both bodies follow elliptical orbits around the system's centre of mass. The semi-major axis of the star's orbit is

$a_{*}=a_{\mathrm{p}} M_{\mathrm{p}} / M_{*}$,

where $a_{\mathrm{p}}$ is the semi-major axis of the planet orbit, $M_{\mathrm{p}}$ is the planet mass, and $M_{*}$ is the stellar mass. The astrometric signature $^{1} v$ is the angular size of the stellar orbit

$v=\left(\frac{M_{\mathrm{p}}}{M_{*}}\right)\left(\frac{a_{\mathrm{p}}}{\mathrm{au}}\right)\left(\frac{d}{\mathrm{pc}}\right)^{-1} \quad \operatorname{arcsec}$,

where $d$ is the distance from the observer to the planetary system.

The orbital elements define the orbit on the true plane. Three of them are called dynamical elements as they define the motion in the orbit: the period $P$, the time of periastron passage $T_{\mathrm{p}}$, and the eccentricity $e$. Four geometrical elements describe the size of the orbit and its orientation with respect to the plane of the sky: the semi-major axis $a_{*}$, the longitude of the ascending node $\Omega$, the argument of periapsis $\omega$, and the inclination $i$ (see Fig. 1).

\footnotetext{
1 From the Greek $v \pi о \gamma \rho \alpha \phi \eta$ (signature).
}

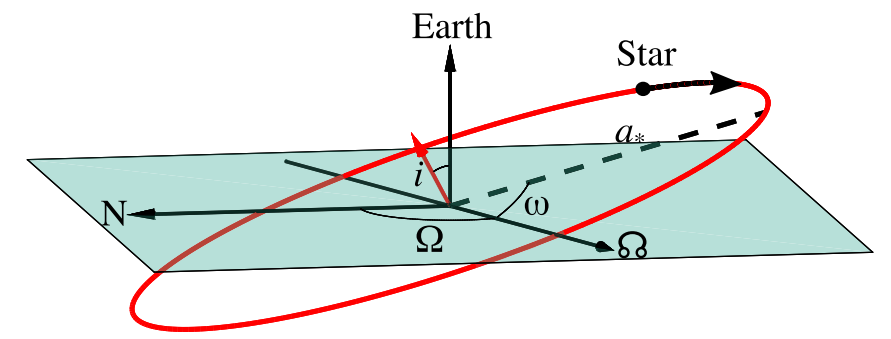

Fig. 1. Illustration of the four geometrical elements: the semi-major axis $a_{*}$, the longitude of the ascending node $\Omega$ (also known as receding node), the inclination $i$, and the argument of periapsis $\omega$. The light cyan polygon represents the sky plane in a perspective view; the reference direction is taken towards the north $(\mathrm{N})$. The line of nodes, shown by the black arrow pointing towards the ascending node, is the intersection between the sky and the orbit planes. The orbit is shown as the red ellipse. The small red arrow is perpendicular to the true plane and shows the inclination.

The instantaneous position on the sky of a star at time $t$ is determined by a fixed displacement $\left(\Delta \alpha_{0}, \Delta \delta_{0}\right)$ from its nominal position $\left(\alpha_{0}, \delta_{0}\right)$ at a reference time $t_{0}$, the proper motion vector ${ }^{2}$ $\left(\mu_{\alpha}, \mu_{\delta}\right)$, the parallax $\varpi$, and the orbital elements. In Cartesian coordinates lying on a plane tangent to the sky and directed along right ascension and declination, respectively (Michalik et al. 2014), the position can be written as

$$
\begin{aligned}
& \Delta \alpha(t)=\Delta \alpha_{0}+\Pi_{\alpha} \varpi+\left(t-t_{0}\right) \mu_{a}+B X(t)+G Y(t), \\
& \Delta \delta(t)=\Delta \delta_{0}+\Pi_{\delta} \varpi+\left(t-t_{0}\right) \mu_{\delta}+A X(t)+F Y(t),
\end{aligned}
$$

where $\Pi_{\alpha}$ and $\Pi_{\delta}$ are the components of the parallax direction, $X$ and $Y$ are the displacement per unit amplitude due to planetary motion in the true plane projected onto the sky through the Thiele-Innes (TI) constants, also called natural elements $A, B, F, G$ (Thiele 1883; van den Bos 1926, 1932):

$$
\begin{aligned}
A & =v(\cos \omega \cos \Omega-\sin \omega \sin \Omega \cos i) \\
B & =v(\cos \omega \sin \Omega+\sin \omega \cos \Omega \cos i) \\
F & =v(-\sin \omega \cos \Omega-\cos \omega \sin \Omega \cos i) \\
G & =v(-\sin \omega \sin \Omega+\cos \omega \cos \Omega \cos i) .
\end{aligned}
$$

In the true plane the coordinates $X$ and $Y$ depend on the eccentric anomaly $E$ and on the eccentricity $e$ :

$$
\begin{aligned}
& X(t)=\cos E(t)-e \\
& Y(t)=\sqrt{1-e^{2}} \sin E(t) .
\end{aligned}
$$

The eccentric anomaly is obtained from Kepler's equation

$$
E(t)=M(t)+e \sin E(t),
$$

where $M$ is the mean anomaly:

$M(t)=\frac{360^{\circ}}{P}\left(t-T_{\mathrm{p}}\right)$.

Within the TI formalism, the determination of the orbital elements from astrometric observations is a non-linear problem in $P, e$, and $T_{\mathrm{p}}$, and linear in all other variables.

2 The proper motion component in the right ascension includes the $\cos \delta$ factor, and corresponds to the $\mu_{\alpha^{*}}$ in Appendix A of Michalik et al. (2014). 


\subsection{Degeneracies}

In some cases, some parameters can become degenerate. For instance, in the case of circular orbits, the argument of periapsis $\omega$ loses its geometrical meaning as direction of the semi-major axis of the orbital ellipse, and it becomes degenerate with the periapsis transit time $T_{\mathrm{p}}$.

A second case is that of face-on orbits, where $\cos i= \pm 1$. Here, the true plane of the orbit coincides with the sky plane, so that both the longitude of the ascending node $\Omega$ and the argument of periapsis $\omega$ are measured on the same plane. The intersection of the two planes, which defines the ascending node, becomes undefined. If Eqs. (5)-(8) are left unchanged, then the sum $\Omega+\omega$ identifies the reference direction from which the time of periastron passage $T_{\mathrm{p}}$ is measured, but $\Omega$ and $\omega$ themselves become undefined. A possible change to the model could be to use a different definition for $\omega$ (e.g. measure it from the north direction).

A third case happens because astrometric data cannot distinguish the ascending node from the descending one. In principle, both the longitude of the ascending node $\Omega$ and the argument of periapsis $\omega$ vary between $-180^{\circ}$ and $180^{\circ}$. However, the same projected orbit can be obtained by adding $180^{\circ}$ to both $\Omega$ and $\omega$, i.e. by exchanging the ascending node for the descending node and the periapsis for the apoapsis. It is customary when analysing astrometric data to restrict the allowed range of $\Omega$ to the $\left[0^{\circ}, 180^{\circ}\right]$ interval (Binnendijk 1960; Perryman et al. 2014). This degeneracy could be broken if one were to jointly analyse astrometric and radial-velocity data.

The above degeneracies are observed in our analysis and the affected systems will be identified in Sect. 6.

\section{Detectability as a function of the $S / N$}

Previous works by $\mathrm{C} 08$ and P14 have shown that the main parameters, upon which the detectability of a planet depends, are the period $P$ and the $\mathrm{S} / \mathrm{N}$

$S / N=\frac{v}{\sigma_{\mathrm{AL}}}$,

where $v$ is the astrometric signature and $\sigma_{\mathrm{AL}}$ is the single-scan uncertainty on our observable, the shift in the along-scan (AL) field angle $\eta$ (defined in Sect. 4.1).

Detectability may also depend on the period because orbit sampling becomes difficult or incomplete either if $P$ is shorter than the average time between two Gaia scans, or if $P$ is longer than the mission length. This is explored in Sect. 7.

Any value of $S / N$ can be mapped, using Eq. (2), to a subspace of $M_{\mathrm{p}}, M_{*}, a_{\mathrm{p}}, d$, and of the stellar magnitude $V$, which would produce the same simulated data. This is illustrated for a few example cases in Fig. 2, where planets of different masses (1 Neptune mass, 1 Jupiter mass, and 13 Jupiter masses ${ }^{3}$ ) are assumed to orbit a bright star at different semi-major axes and distances. Both $\mathrm{C} 08$ and P14 suggested that a $S / N \gtrsim 3$ is needed to detect a planet; therefore, Fig. 2 also shows an approximate estimate of the distance from Earth up to which a planet can be detected.

Therefore, in the following we will parameterise our simulations over a grid of $P$ and $S / N$. Only when relevant do we trace the $S / N$ back to the parameters on which it depends.

\footnotetext{
3 A mass of $13 M_{\text {Jup }}$ is the approximate limit mass for deuterium burning, and it is usually taken as the upper limit for planetary masses (Boss et al. 2007).
}

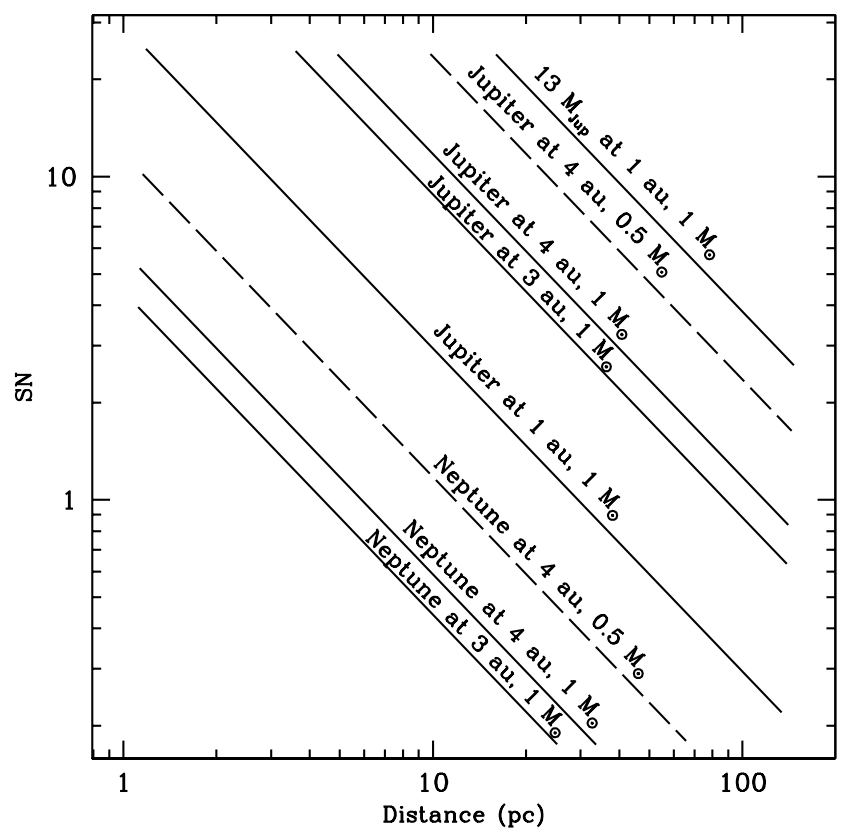

Fig. 2. Expected (S/N) for different planet masses as a function of distance. The lines show the cases for 13 Jupiter masses, 1 Jupiter mass, and 1 Neptune mass; with circular orbits at 1, 3, and 4 au (it is only possible to observe a 4 au orbit if the mission length is $\sim 10 \mathrm{yr}$; see Sect. 8). A $1 M_{\odot}$ star is assumed for the solid lines, while a $0.5 M_{\odot}$ star is assumed for the dashed lines.

\section{Simulated Gaia observations}

\subsection{Field angles in the Gaia field of view}

The Gaia spacecraft scans the sky along great circles whose orientation changes in time, following a complex pattern which we refer to as the Gaia scanning law. For each star transiting any of the two fields of view, the time-dependent position in the AL direction is measured on the sky mapper charge-coupled devices (CCD) and on each of the nine astrometric field CCDs. The formal uncertainty $\sigma_{\mathrm{AL}}$ results from combining all ten measurements, and it depends on the stellar magnitude, with $\sigma_{\mathrm{AL}}=$ 0.034 mas for $V \leq 14$ for a single scan, and larger for fainter stars (de Bruijne 2012; de Bruijne et al. 2014). A coarser determination of the position in the across-scan (AC) direction is also done, but issues of pixel size and binning make $\mathrm{AC}$ measurements less useful for astrometry. Gaia returns on average 70 times to each star during its 5 yr mission (de Bruijne et al. 2010), and each time the transit in the field-of-view measurement occurs with a different orientation, thus the stellar position can be pinpointed with AL information only.

If $\theta(t)$ is the position angle of the Gaia field of view, the shift in the AL field angle $\eta$ is given by

$\Delta \eta(t)=\Delta \alpha \cos \theta(t)+\Delta \delta \sin \theta(t)$

and Eqs. (3) and (4) can be expressed in terms of $\mathrm{AL}$ displacement:

$$
\begin{aligned}
\Delta \eta(t)= & \Delta \alpha_{0} \cos \theta(t)+\Delta \delta_{0} \sin \theta(t) \\
& +\Pi_{\alpha} \varpi \cos \theta(t)+\Pi_{\delta} \varpi \sin \theta(t) \\
& +\left(t-t_{0}\right) \mu_{\alpha} \cos \theta(t)+\left(t-t_{0}\right) \mu_{\delta} \sin \theta(t) \\
& +(B X(t)+G Y(t)) \cos \theta(t) \\
& +(A X(t)+F Y(t)) \sin \theta(t)
\end{aligned}
$$


Table 1. Probability distributions of randomised parameters for the single-planet simulations.

\begin{tabular}{|c|c|}
\hline Parameter & Distribution \\
\hline RA, Dec & Uniform over the celestial sphere \\
\hline$\Delta \alpha_{0}$ (mas) & Normal $(0,0.04)$ \\
\hline$\Delta \delta_{0}$ (mas) & Normal $(0,0.04)$ \\
\hline$d(\mathrm{pc})$ & $\log$-normal $\left(\ln \frac{1 \mathrm{mas}}{\sigma_{\Delta N} S / N}, 0.6\right)$ \\
\hline$\varpi(\mathrm{mas})$ & $1000 / \mathrm{d}$ \\
\hline$\mu_{\alpha}, \mu_{\delta}\left(\operatorname{mas~yr}^{-1}\right)$ & $\begin{array}{l}\text { taken randomly from Gaia DR1 } \\
\text { stars with distance } \sim d\end{array}$ \\
\hline$T_{\mathrm{p}}(\mathrm{yr})$ & Uniform $(0, P)$ \\
\hline$\Omega$ & Uniform $\left(0,180^{\circ}\right)$ \\
\hline$\omega$ & Uniform $\left(-180^{\circ}, 180^{\circ}\right)$ \\
\hline $\cos i$ & Uniform $(-1,1)$ \\
\hline
\end{tabular}

Notes. The parameters for the normal distribution are mean and sigma; for the log-normal they are log-mean and sigma; for the uniform they are the bounds of the allowed interval.

\subsection{Single-planet systems: simulations over a grid of parameters}

Ideally, we aim to test how the detection methods perform over the possible range of variation of all parameters. However, the high dimensionality of the problem of determining astrometric and Keplerian parameters together prevents a full exploration of all parameters on a grid.

For our single-planet simulations, we selected three parameters to be explored on a grid:

- the $\mathrm{S} / \mathrm{N}$;

- the period $P$;

- the eccentricity $e$.

All the other parameters were randomised using the probability distributions listed in Table 1 and explained in Sect. 4.3.

We produced a first batch of simulations for a $5 \mathrm{yr}$ mission (the nominal Gaia mission length), and a second batch for a $10 \mathrm{yr}$ mission to investigate the improvements in case the mission is extended. In both batches, our grids ran with logarithmic increments over the ranges $S / N=0.2-10, P=0.08-10 \mathrm{yr}$, and $e=0.01-0.97$. To smooth the grid and make the plots more readable, we added small random amounts to the $\mathrm{S} / \mathrm{N}, P$, and $e$ of each simulation. The upper bound to the eccentricity corresponds to an ellipse with a 1:4 ratio between the semi-minor and semi-major axis.

\subsection{Randomised astrometric parameters}

We set the RA and Dec coordinates of the host star to random values, uniformly distributed over the celestial sphere. The Gaia scanning law was computed for each position using the AGISLab software (Holl et al. 2012), which was developed as part of the Gaia astrometric global iterative solution (AGIS; Lindegren et al. 2012) and is available to users within the Gaia Data Processing and Analysis Consortium (DPAC).

We set the astrometric parameters $\left(\Delta \alpha, \Delta \delta, \varpi, \mu_{\alpha}, \mu_{\delta}\right)$ to random values according to the probability distributions listed in Table 1 . We assumed $\Delta \alpha$ and $\Delta \delta$ to be normally distributed, with a standard deviation of 1 mas. The remaining parameters depend on the distance $d$, which was assumed to be log-normally distributed around a value which depends on the $\mathrm{S} / \mathrm{N}$. The range of values covered by $d$ was chosen so that the $\mathrm{S} / \mathrm{N}$ could include the cases of a Jupiter-mass planet at 3 au around a $1 M_{\odot}$ star, and of a Neptune-mass planet at 4 au around a $0.5 M_{\odot}$ star. The parallax was obtained from the distance. The proper motions should reflect the larger spread in velocities of nearby stars with respect to distant stars; therefore, we drew proper motions from the Gaia data release 1 (DR1; Gaia Collaboration 2016a; Lindegren et al. 2016).

We set the remaining orbital elements $\left(T_{\mathrm{p}}, \Omega, \omega, i\right)$ to random values, different for each simulation, uniformly distributed over their natural ranges of variation: $0 \leq T_{\mathrm{p}}<P, 0 \leq \Omega<180^{\circ}$, $-180^{\circ} \leq \omega<180^{\circ},-1 \leq \cos i \leq 1$.

Kepler's equation needs to be solved for every simulated Gaia scan; we used the numeric solver vpasolve in the MATLAB package, which provides solutions with double-precision accuracy ${ }^{4}$.

\subsection{Simulations of systems with no planet}

To help set up the thresholds for planet detection, we also simulated no-planet systems. These systems follow the same specifications of the previous sections for the astrometric parameters, noise, and scanning law, but no Keplerian signal is added.

\section{Bayesian analysis}

In this section, we assume some familiarity with Bayesian inference and its nomenclature (prior, likelihood, posterior, Markov chains, etc). The interested reader can find an introduction geared towards astrophysics in Trotta (2008) or a more general discussion in the textbook by Gregory (2005).

\subsection{Modelling}

From a model point of view, Bayesian inference works similarly to maximum likelihood in that a series of tentative sets of parameters are generated, and each set is used to simulate an observable quantity which is then compared against the data. In our setting the data are $\eta_{0, s}$, i.e. the observed AL displacements, one per Gaia scan $s$, computed at simulation time using Eq. (15). Let $\eta_{i, s}$ be the displacements computed during the fit for a set $i$ of astrometric and orbital parameters. The likelihood can then be obtained by assuming that the difference $\eta_{0, s}-\eta_{i, s}$ is normally distributed, with mean zero and standard deviation $\sigma_{\mathrm{AL}}$ :

$$
\mathcal{L}(i)=\frac{1}{\sqrt{2 \pi} \sigma_{\mathrm{AL}}} \prod_{s} \mathrm{e}^{-\frac{1}{2} \frac{\left(\eta_{0, s}-\eta_{i, s}\right)^{2}}{\sigma_{\mathrm{AL}}}}
$$

We used the Stan package for statistical inference (Carpenter et al. 2016, version 2.12) to model our simulated Gaia observations. The Stan package allows models to be specified in a programming language very similar to that of popular packages such as BUGS (Lunn et al. 2000) or JAGS (Plummer et al. 2003). All these packages implement general-purpose MCMC samplers together with a high-level programming language to specify the model.

Our implementation follows the formalism of Sect. 2.1. For each simulated system, we define two models:

- a purely astrometric model, which only contains $\Delta \alpha_{0}, \Delta \delta$, $\varpi, \mu_{\alpha}$, and $\mu_{\delta}$. This model can be obtained by setting $A=B=F=G=0$ in Eq. (15). This model is the baseline

4 We also experimented with the Mikkola (1987) cubic approximation, which has a relative error within $0.2 \%$. We found that the use of the approximation was hindering the successful fit of the simulations with the method described in Sect. 5. 
Table 2. Priors for the purely astrometric model.

\begin{tabular}{ll}
\hline \hline Parameter & Distribution \\
\hline$\Delta \alpha_{0}(\mathrm{mas})$ & Normal $(0,0.04)$ \\
$\Delta \delta_{0}(\mathrm{mas})$ & Normal $(0,0.04)$ \\
$\varpi(\mathrm{mas})$ & Normal $(2.8,59) ; \varpi \geq 0$ \\
$\mu_{\alpha}\left(\mathrm{mas} \mathrm{yr}^{-1}\right)$ & Normal $\left(0,9.5 \varpi^{0.89}\right)$ \\
$\mu_{\delta}\left(\mathrm{mas} \mathrm{yr}^{-1}\right)$ & Normal $\left(0,10 \varpi^{0.83}\right)$ \\
\hline
\end{tabular}

Notes. The parallax $(\varpi)$ has the additional constraint that it must be non-negative. Proper motions have a parallax-dependent standard deviation. Distribution parameters as in Table 1.

against which the full model is compared when evaluating a detection;

- a full model, containing all the parameters of Eq. (15).

Kepler's equation needs to be solved at each MCMC iteration, but Stan does not provide a solver in its current version; therefore, we use the Markley (1995) approximation, which is fast to compute and provides, in our implementation, a relative error of the order of $10^{-14}$, close to the limits of a double-precision variable.

\subsection{Model priors}

\subsubsection{Purely astrometric model}

The prior probability distributions for the model parameters should be set according to the expected range of variation, and to the expected distribution for their values.

For the astrometric model we chose very weakly informative priors which only set the physical scale of the parameters (Table 2). For $\Delta \alpha_{0}$ and $\Delta \delta$ we used normal distributions with mean 0 and standard deviation 0.04 mas, consistently with the expected perturbation induced by a Jupiter-mass planet. For the proper motions $\mu_{\alpha}$ and $\mu_{\delta}$ we used normal distributions with mean 0 and standard deviation that depends on the parallax to reproduce the fact that more distant stars have lower velocities. By fitting the Gaia DR1 stars within $\sim 200$ pc, we found $\sigma_{\alpha_{0}} \propto \varpi^{0.83}$ and $\sigma_{\delta} \propto \varpi^{0.89}$. For the parallax $\varpi$, we used a lognormal prior (with $\ln ($ mean $)=2.8$, corresponding to 16 mas, and $\ln$ (standard deviation $)=7$ ) which we found to reasonably reproduce the HIPPARCOS and Gaia DR1 data. We also added the constraint that $\varpi \geq 0$.

\subsubsection{Full model}

For some parameters $\left(\Omega, \omega, \cos i\right.$, and $\left.T_{\mathrm{p}}\right)$ the obvious choice is to set the priors to constant distributions according to the parameter range (see Table 3). A uniform distribution is therefore our choice for $\cos i$. However, $\Omega$ and $\omega$ are angles, and $T_{\mathrm{p}}$ can also be treated as such, thus one needs to ensure that the prior distribution is defined over a circular support so as not to introduce any artificial barrier for when the Markov chains explore the likelihood space. This is achieved by a von Mises distribution, with concentration parameter $\kappa \sim 0$, which is the circular equivalent of a uniform distribution ${ }^{5}$ (Jammalamadaka \& SenGupta 2001).

5 The von Mises distribution is a circular analogue of a normal distribution. It is a continuous probability distribution whose support is a circle, and it is defined as von Mises $\left(x ; \mu_{\mathrm{vM}}, \kappa\right)=\frac{e^{\kappa \cos \left(x-\mu_{\mathrm{VM}}\right)}}{2 \pi I_{0}(\kappa)}$, where $I_{0}(\kappa)$ is the modified Bessel function of order 0 . The parameters are the location of its peak $\mu_{\mathrm{vM}}$ and the concentration $\kappa$ (analogous to
Table 3. Priors for the orbital elements of the planet.

\begin{tabular}{ll}
\hline \hline Parameter & Distribution \\
\hline$P(\mathrm{yr})$ & $\ln (P / 1 \mathrm{yr})$ is uniform $(-6,5)$ \\
$v(\mathrm{mas})$ & $\log$-normal $(-2,1)$ \\
$\Omega$ & von Mises $\left(0,0.001\right.$, support $\left.=\left[0,180^{\circ}\right]\right)$ \\
$\omega$ & von Mises $\left(0,0.001,\left[-180^{\circ}, 180^{\circ}\right]\right)$ \\
$\cos i$ & Uniform $(-1,1)$ \\
$e$ & Uniform $(0,0.9999)$ \\
$T_{\mathrm{p}}(\mathrm{yr})$ & von Mises $(0,0.001,[0, P])$ \\
\hline
\end{tabular}

Notes. The full model includes both the orbital elements and the astrometric parameters listed in Table 2. The parameters for the von Mises distribution are location and concentration $(\kappa)$. Other distributions are as in Table 1 . The eccentricity is bound to the [0, 0.9999] interval whose upper bound corresponds to an ellipse with a 1:70 ratio between the semi-minor and semi-major axes.

For the other orbital parameters we chose non-informative priors as follows:

- For $P$, we considered a prior where $\ln P$ is uniformly distributed, so that no preference for any scale is introduced. Periods shorter than 1 day or longer than $150 \mathrm{yr}$ were excluded.

- For $e$, we also considered a uniform prior. An upper bound $e=1$ cannot be used because it corresponds to an open orbit. Therefore, we took $e=0.9999$ as the upper bound (which corresponds to an ellipse with a ratio between the major and minor axes of $\sim 70$ ).

- For the angular size of the semi-major axis $v$ we chose a $\log$-normal distribution with $\ln ($ mean $)=-2$ (corresponding to 0.14 mas) and $\ln$ (standard deviation) $=1$, with an upper bound at 10 mas. This distribution puts $90 \%$ of the probability on systems with $v<0.5$ mas, yet it allows for some rarer systems closer to Earth where wider angular orbits might be expected.

Finally, astrometric parameters in the full model have the same priors as in the purely astrometric model.

\subsection{Chain initialisation}

Markov chains (MC) need a starting point. From there, the MC will start to explore the parameter space. If the starting point is not in a high-probability region, it is necessary to discard the first iterations (usually called the burn-in period or warm-up period) to ensure that the rest of the chain represents a sample of the posterior distribution, with no bias due to the presence of many initial steps exploring a low-probability region.

In principle, the starting point can be taken randomly, though this may result in a very long burn-in period. Therefore, statistical literature often suggests starting the Markov chains at a point reasonably close to the likelihood global maximum if it is known (e.g. Geyer 2011).

Our tests have shown that the MC burn-in can be prohibitively long for the needs of a survey unless a starting point close to the correct values for the parameters is used. However, in our case the structure of the likelihood space presents many

the inverse of the variance of a normal; distributions with higher $\kappa$ have sharper peaks). When $\kappa=0$, no peak is present and the location becomes undefined. Ideally, one should set $\kappa=0$; however, the current Stan implementation of the von Mises distribution is restricted to $\kappa>0$, so we chose a $\kappa$ low enough to be practically indistinguishable from 0 . 
very narrow peaks ${ }^{6}$, which prevent common optimisation methods (e.g. Amoeba, Levenberg-Marquardt, or Broyden-FletcherGoldfarb-Shanno) from finding the global likelihood maximum.

We have considered two possibilities for the starting point: first, a perfect starting point corresponding to the true value of the parameters; second, a best-fit point obtained from a simplified model that assumes circular orbits. The first option is motivated by our aim to test our Bayesian model and check how it behaves in an ideal case. The second option is more realistic; our motivation is to test whether a very simple and fast model can be used as a reasonable starting point or if a more refined approximation is needed. In the reality of any future survey using Gaia data, the first option will of course be unavailable, while more refined methods used to find a starting point can be used (e.g. Sahlmann et al. 2013).

In the circular orbits model, the period $P$ is the only parameter which appears non-linearly. We defined a grid of frequencies $f=1 / P$, starting from $f=0.067$ and increasing to $12.5 \mathrm{yr}^{-1}$, with a step of $0.03 \mathrm{yr}^{-1}$, covering the allowed interval for $P$. For every value of $f$, we obtained the best-fit values for all other parameters and the $\chi^{2}$ using linear least-squares. The values which scored the lowest $\chi^{2}$ provided the starting point for the MC.

The circular-orbit model only includes ten parameters: $e$ does not appear, and $\omega$ and $T_{\mathrm{p}}$ become degenerate, so that only one of them is needed. To make our implementation easier, we dropped $T_{\mathrm{p}}$ and retained $\omega$. Therefore, we were left with the need to specify starting values for $e$ and $T_{\mathrm{p}}$. We chose $e=0.1$ and $T_{\mathrm{p}}=0$.

\subsection{Chain convergence}

In the long run, the set of points touched by a MC forms a non-biased sample of the target probability distribution; when this happens, it is said that the MC has converged to the target. The main risk associated with non-converging chains is that, since the MCMC samples would not be representative of the posterior distribution, any inference on the model parameters might be severely biased. The purpose of the burn-in period is to assure that the chains have converged before posterior samples are obtained from them. However, there is little guidance on how long the burn-in period should be, so one has to rely on experience and on inspection of the MCMC samples.

We initially conduced some test runs of our Stan implementation to understand the number of iterations needed for the Markov chains burn-in period. Visual inspection of the chain traces showed that the burn-in period lasted less than a few hundred iterations in many cases where $S / N \gtrsim 3-5$, but in some cases it could be considerably longer.

Therefore, for the simulations described in Sect. 4.2, we initially decided to use a burn-in period of 500 iterations. Then we checked whether the chain had converged after this period, or if more burn-in iterations were needed, according to the procedure described as follows.

After the 500 burn-in iterations, we collected the next 500 iterations for further analysis. On the latter samples we computed for each parameter $i$ the potential scale reduction $\hat{R}_{i}$, which measures how much sharper an estimate might become if the MCs were run indefinitely (Gelman \& Rubin 1992; Eq. (11.4) in Gelman et al. 2013). The $\hat{R}$ compares the variance among the

6 The highest secondary peaks are randomly located and do not seem to be aliases of the true period; there are usually only a few for very high $\mathrm{S} / \mathrm{N}$ (e.g. $S / N \sim 10$ ). In addition, a large number of lower peaks are present even at $S / N \sim 0$, which can just be attributed to noise. The peak narrowness does not depend on the $\mathrm{S} / \mathrm{N}$. chains versus the variance within a single chain: if all chains have converged and are sampling the same space, both variances should be approximately equal, and $\hat{R} \sim 1$. A value of $\hat{R} \gtrsim 1.1$ is usually considered as pointing towards convergence problems. The opposite is not necessarily true (it is possible to have $\hat{R}<$ 1.1 and still have convergence problems) because convergence can never be guaranteed when the number of samples is finite, though in our experience the $\hat{R}$ criterion has matched very well any conclusion that could be made based on visual inspection.

We found that, for any given simulated system, the $\hat{R}_{i}$ can vary among the parameters, with some of them showing convergence problems, while other seem to be fine. Therefore, we also computed the average $\hat{R}_{\text {avg }}=\frac{1}{N} \sum_{i=1}^{N} \hat{R}_{i}$. Whenever a simulated system was found to have $\hat{R}_{\text {avg }}>1.1$, we discarded its samples and we ran the chains anew, this time with 15000 burn-in iterations.

After that, if $\hat{R}_{\text {avg }}$ was still unsatisfying, we deemed the chains as not converging and excluded the planet from further consideration.

There may be different reasons for not fulfilling the convergence criterion, some of which do not prevent meaningful information to be retrieved. However, it would be difficult to express how confident one could be in any estimate coming from non-converging chains. Therefore, in the context of a blind survey of exoplanets, information from non-converging chains might be of little use; in the context of extracting the most information from Gaia data of a particular planet (say, by joint fitting of astrometric and radial velocity, or transit data) there would probably be more prior information to help find a different (perhaps better) starting point for the chain.

\subsection{Exoplanet detection as model selection}

Deciding whether an exoplanet has been detected is essentially a comparison of model performances. The null model is that no planet is present, and that the Gaia measurements can be adequately explained with just the astrometric parameters. The alternative model is that a planet is present, and both the astrometric and Keplerian parameters are needed.

The classical (frequentist) approach is to use hypothesis testing: the difference between the models' $\chi^{2}$ is computed and linked, through the number of degrees of freedom (dof), to a $p$-value. It can be applied to the case of exoplanet detection, provided that the Thiele-Innes parameterisation is adopted. One would therefore obtain the $A, B, F$, and $G$ parameters from the fit, and only later would they be reconnected to the orbital elements $v, \omega, \Omega$, and $\cos i$. Uncertainties on the best-fit values should then be propagated from the Thiele-Innes parameters back to the orbital elements.

However, a complication is that the number of dof is unambiguously defined only for linear models. Non-linear models behave as if they have a larger number of dof than a linear model with the same number of parameters. Also, the "effective dof" of a non-linear model are data-dependent (through the variance of the parameters; see e.g. Eq. (19) below), and they are larger when the data are noisy (Andrae et al. 2010); we will show this happening in our simulations in Sect. 6.2.

Conversely, the Bayesian approach has the following advantages: the orbital elements can be the subject of inference, the uncertainties on the fit parameters need not be estimated using asymptotic theory, and there are model comparison methods which explicitly account for the effective dof.

An easy way to perform Bayesian model comparison is to use information criteria (IC) because only the posterior samples 
are needed for their calculation. The theory of most IC is based on the notion of predictive accuracy, i.e. how well a model fit can predict new data produced from the true data-generating process. A notable exception is the Bayesian information criterion (BIC, see below) which is motivated by the marginal probability of the data under the model. A introduction to both criteria from an astrophysics perspective can be found in Liddle (2007); among the statistical sources, we refer to Gelman et al. (2013, Chap. 7) and Burnham \& Anderson (2002).

The IC operate in practice by computing a function of the likelihood, either at a specified point or averaged over the posterior. The function includes a penalty, based on the number of dof, to account for overfitting by the more complex model.

Some IC also find application in the context of frequentist statistics as they can be applied to maximum likelihood estimates. Therefore some of our results might also find application in future work that does not use the Bayesian framework.

The best known IC are the Akaike information criterion (AIC; Akaike 1973),

$\mathrm{AIC}=-2 \ln \mathcal{L}_{\max }+2 k$,

and the Bayesian information criterion (BIC; Schwarz 1978),

$\mathrm{BIC}=-2 \ln \mathcal{L}_{\max }+k \ln n$,

where $\mathcal{L}_{\max }$ is the maximum likelihood, $k$ is the number of free parameters, and $n$ is the number of data points. The main difference is that the penalty on BIC also depends on the number of data points.

Both the AIC and the BIC rely on a point estimate of the model, and they still involve counting the free parameters. A recent development on the AIC is the widely applicable (or Watanabe-Akaike) information criterion (WAIC), which has been proposed as a fully Bayesian approach of estimating the predictive accuracy (Watanabe 2010; see Ranalli et al. 2016 for an application to astrophysics). It uses all available samples of the posterior distribution and gets the effective dof from the parameter variance:

$$
\begin{aligned}
\text { WAIC }= & -2 \ln \left(\frac{1}{I} \sum_{i=1}^{I} \mathcal{L}(i)\right) \\
& +2 \sum_{s=1}^{n} \frac{1}{I-1} \sum_{i=1}^{I}\left(\ln \mathcal{L}(i, s)-\langle\ln \mathcal{L}(i, s)\rangle_{i}\right)^{2} .
\end{aligned}
$$

Here, the index $i$ runs on the samples from the posterior distribution, and the index $s$ runs on the data points (the Gaia scans), so both indices keep the same meaning they had in Eq. (16). The symbol $\mathcal{L}(i, s)$ is the likelihood of a single data point (scan). The angular brackets with the $i$ subscript indicate that the average over the samples should be taken; the squared difference is therefore the variance of the log-likelihood ${ }^{7}$.

In all cases, the lower the IC, the more favoured the model. However, the absolute scale of the IC does not carry statistical meaning; the important quantity is the difference between the IC of different models, not the values of the IC themselves. Therefore, a large $\Delta \mathrm{IC}$ can indicate a strong preference for one model over the other, while a small $\triangle \mathrm{IC}$ indicates

\footnotetext{
7 Watanabe (2013) also proposed an extension of the BIC, the widely applicable Bayesian information criterion (WBIC). However, to compute the WBIC samples from both the posterior and the prior are needed. The latter are not provided by the current Stan version, and therefore we do not consider the WBIC here.
}

that one model does not perform appreciably better than the other.

The $\triangle \mathrm{IC}$ can also be interpreted using Jeffreys' scale of evidence (Efron \& Gous 2001), which rates $\Delta \mathrm{IC} \gtrsim 1,3,20$, and 150 as "barely worth reporting", "weak", "strong", and "very strong", respectively.

The preference can be quantified by considering the relative likelihood $w$ of model $M_{1}$ over model $M_{2}$. Assuming $\operatorname{IC}\left(M_{1}\right)>$ $\mathrm{IC}\left(M_{2}\right)$ (so that $M_{2}$ is the preferred model),

$w=\mathrm{e}^{-\frac{1}{2} \Delta \mathrm{IC}_{12}}$,

where $\Delta \mathrm{IC}_{12}=\mathrm{IC}\left(M_{1}\right)-\mathrm{IC}\left(M_{2}\right)$ (Burnham \& Anderson 2002, see their Sects. 2.8 and 2.9). Strong evidence, in the sense of Jeffreys' scale, would correspond to a relative likelihood of $w \sim$ $4.5 \times 10^{-5}$ for the worse model over the preferred one.

Reflecting the different definitions of the IC, the actual values of $w$ will depend on which IC is considered. The most important factor is the $\ln n$ term that appears in BIC (Eq. (18)), but not in AIC or WAIC (Eqs. (17) and (19)). The BIC in fact has the property that in the limit of large $n$ the penalty tends to infinity. The AIC and WAIC do not have this property.

The importance of this property is more evident if one considers the case of a real multiplanet system whose data are fit by a set of models that approximate our physics knowledge better and better (e.g. no planet; one planet; two planets ignoring their mutual attraction; two planets including their mutual attraction; three planets, etc.). Obviously, some models are more easily distinguished (no planet vs. one planet), while some others are more difficult to distinguish (mutual attractions). In the limit of large $n$, BIC will discard models that overfit data more easily than AIC or WAIC, while AIC and WAIC will instead identify a subset of similarly behaving models. Whether this property of BIC is desirable depends on whether one expects that the "true" model belongs in the considered set. One seldom or probably never has such an expectation in general. However, planetary motions may represent a niche case where one can expect to include a model that, for a given data quality, is indistinguishable from one that includes all known physical effects.

For Gaia data, $n=73 \pm 23$ and $147 \pm 46$ for the $5 \mathrm{yr}$ and $10 \mathrm{yr}$ mission, respectively, which means that from Eqs. (17) and (18) one expects an average $\triangle \mathrm{AIC}-\triangle \mathrm{BIC} \sim 16$ and 21 , respectively. These values are close to the threshold for strong evidence on Jeffreys' scale. Therefore, we expect that different IC may yield different fractions of false positives if the strong evidence threshold is used. From the defining equations of the IC and for systems with $S / N \sim 0$, we expect that the BIC signals a preference for the simpler model (the astrometric model), while the AIC and WAIC signal that the more complex model (the planetary model) does not offer an advantage over the simpler model.

One might ask what advantage the IC offer over a $\Delta \chi^{2}$ criterion. In a non-linear setting, because of the difficulties in determining the number of dof, a $\Delta \chi^{2}$ is equivalent to an information criterion where one does not know beforehand what penalty to apply to correct for overfitting. It would still be possible to use the $\Delta \chi^{2}$ for model choice after calibrating a threshold based on an allowed fraction of false positives.

\section{Fraction of false-positive detections}

In this section we apply all criteria to our no-planet simulations, so we expect that the purely astrometric model should be preferred in all cases. Our samples consist of 6644 and 4402 no-planet systems for the $5 \mathrm{yr}$ and $10 \mathrm{yr}$ missions, respectively. 

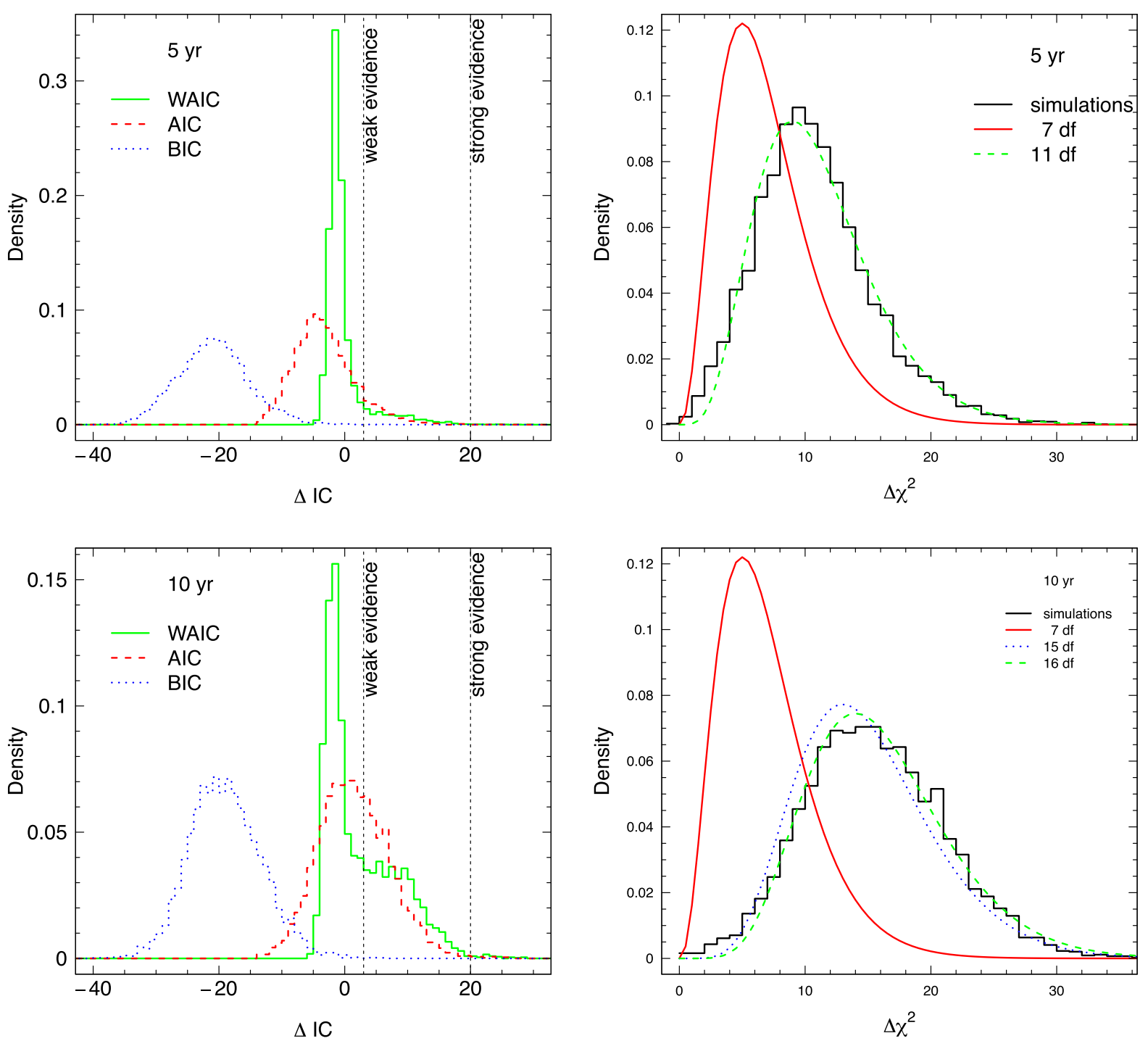

Fig. 3. Histograms for the IC (left panels) and for $\Delta \chi^{2}$ (right panels) in the case of no-planet simulations. The upper panels consider the 5 yr mission, the lower panels the $10 \mathrm{yr}$ mission. Left panels: green solid lines, WAIC; red dashed lines, AIC; blue dotted lines, BIC; the vertical dashed lines show the IC thresholds for weak and strong evidence according to Jeffreys' scale. Right panels: solid and dashed curves show $\chi^{2}$ distributions with the dof reported in the legend. The empirical density does not follow a $\chi^{2}$ distribution with the nominal number of dof (7, solid red line). It can however be approximated, by a $\chi^{2}$ distribution with a larger number of dof: 11 dof for the $5 \mathrm{yr}$ mission, 15 dof (or 16 dof for $\Delta \chi^{2} \geq 30$ ) for the $10 \mathrm{yr}$ mission.

\subsection{Information criteria}

We define $\Delta \mathrm{IC}=\mathrm{IC}\left(M_{a}\right)-\mathrm{IC}\left(M_{k}\right)$, where $M_{a}$ is the astrometric model, and $M_{k}$ is the model with the Keplerian orbit. With our definition, a positive $\triangle \mathrm{IC}$ indicates a preference for the Keplerian model, and vice versa. Values of $\Delta \mathrm{IC} \sim 0$ indicate no preference for one model over the other; therefore, for reasons of simplicity, the simpler model (i.e. the astrometric model) should be preferred. For $\Delta \mathrm{IC}>0$, we use Jeffreys' scale of evidence to interpret the results, and then we define empirical $\Delta \mathrm{IC}$ thresholds based on the number of false positives.

In Fig. 3 (left panels), we show the histograms of $\triangle \mathrm{IC}$ for the no-planet systems. The peaks of the histograms are safely below the IC $>3$ threshold for weak evidence according to Jeffreys' scale, though both AIC and WAIC have a tail in the weak-evidence zone, especially in the $10 \mathrm{yr}$ mission case. Such a tail may be better seen in the plots of the cumulative distributions (Fig. 4). For the 5 yr mission, the strong evidence threshold (IC $>20)$ is exceeded by AIC in 2 cases $(0.03 \%)$ and by WAIC in 11 cases $(0.17 \%)$ out of 6644 cases; for the $10 \mathrm{yr}$ mission it is exceeded by AIC in 12 cases $(0.27 \%)$ and by WAIC in 25 cases $(0.57 \%)$ out of 4402 . The strong evidence threshold is never exceeded by BIC. We list the $99 \%$ and $99.73 \%$ quantiles in Table 4 . The value for the $99.73 \%$ quantile should be considered as approximate because of the small number of systems whose ICs fall above the quantiles (18 and 12 for the $5 \mathrm{yr}$ and $10 \mathrm{yr}$ mission, respectively). When going from the $5 \mathrm{yr}$ to the $10 \mathrm{yr}$ mission, there is an increase in both quantiles for all ICs and for $\Delta \chi^{2}$.

Compared to $\triangle \mathrm{AIC}$ and $\triangle \mathrm{BIC}, \triangle \mathrm{BIC}$ appears to be negative in almost all cases and therefore indicates a preference for the astrometric model.

\section{2. $\Delta \chi^{2}$}

Considering two nested linear models with a different number of parameters (e.g. the astrometric and the full model), and data distributed according to the null model (in our case, the 

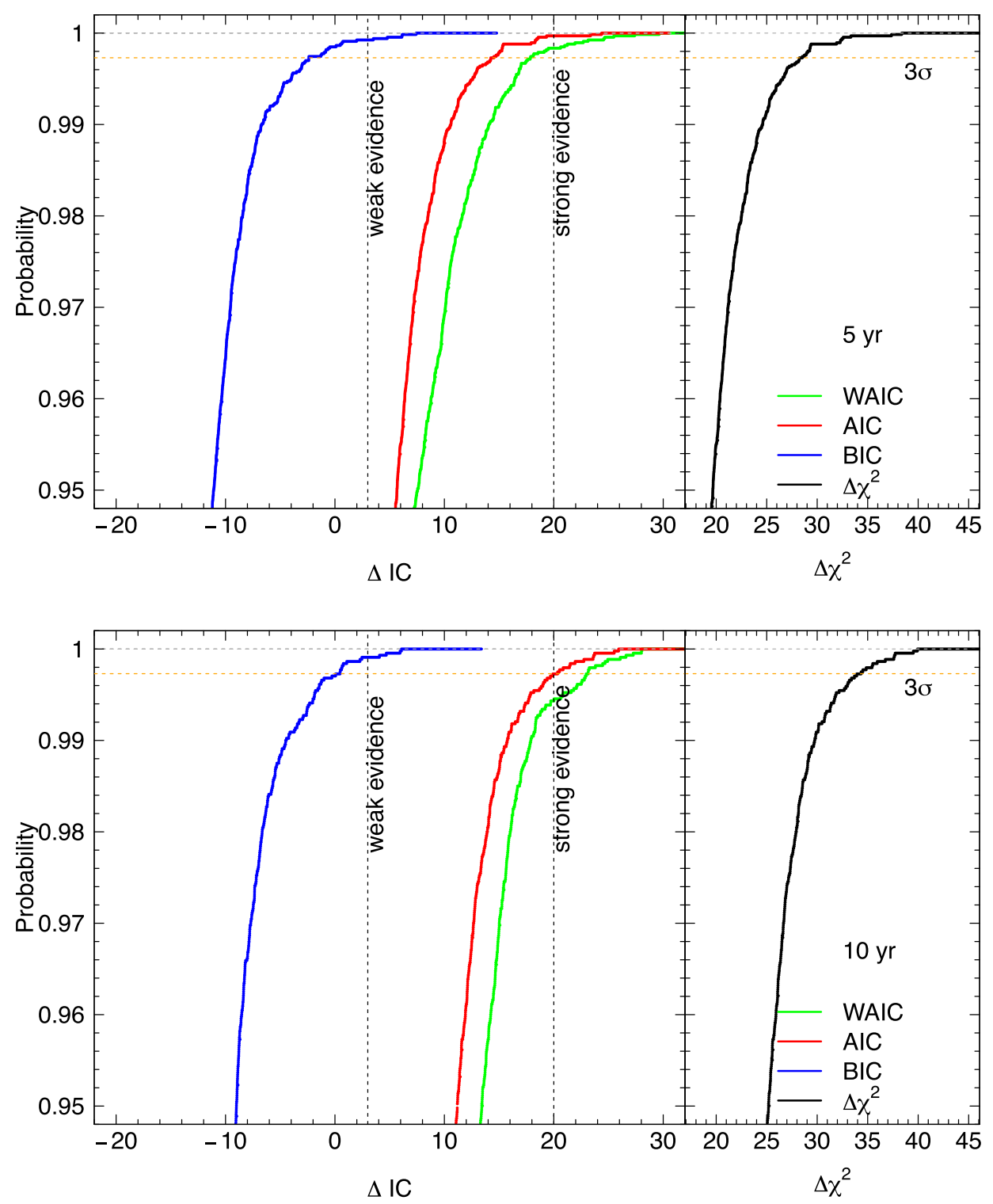

Fig. 4. Cumulative distributions of the IC and $\Delta \chi^{2}$ for no-planet systems in the $5 \mathrm{yr}$ (upper panel) and $10 \mathrm{yr}$ (lower panel) mission simulations. The four curves show (from left to right): BIC (blue), AIC (red), WAIC (green), $\Delta \chi^{2}$ (black). The horizontal dashed lines show the $100 \%$ and $99.73 \%$ $(3 \sigma)$ quantiles. The vertical dashed lines show the IC thresholds for weak and strong evidence according to Jeffreys' scale.
Table 4. Information criteria and $\Delta \chi^{2}$ quantiles for false-positive detections in no-planet simulations, for the four different indicators and for the $5 \mathrm{yr}$ and $10 \mathrm{yr}$ missions.

\begin{tabular}{lrrrr}
\hline \hline \multirow{2}{*}{ Criterion } & \multicolumn{2}{c}{$99 \%$ quantile } & \multicolumn{2}{c}{$99.73 \%$ quantile } \\
\cline { 2 - 5 } & $(5 \mathrm{yr})$ & $(10 \mathrm{yr})$ & $(5 \mathrm{yr})$ & $(10 \mathrm{yr})$ \\
\hline WAIC & 14.0 & 17.9 & 18 & 23 \\
AIC & 10.6 & 15.7 & 14 & 20 \\
BIC & -6.72 & -4.51 & -2.4 & 0.11 \\
$\Delta \chi^{2}$ & 24.6 & 29.7 & 28 & 34 \\
\hline
\end{tabular}

Notes. The first two columns list the $99 \%$ quantile, while the last two columns show the $99.73 \%(3 \sigma)$ quantile values observed. The $99.73 \%$ quantile has been estimated on a small number of objects so its value should be considered approximate.

astrometric model), the difference between the $\chi^{2}$ values of the best fits under the two models still follows a $\chi^{2}$ distribution, with a number of dof equal to the difference between the dof of the two models. As discussed in Sect. 5.5, the dof become ill-defined when the model is non-linear. We show this in Fig. 3 (right panels), where we plot the empirical $\Delta \chi^{2}$ density for the no-planet simulations against a $\chi^{2}$ distribution for seven dof (corresponding to the difference between 12 and 5 parameters in the full and astrometric models, respectively). The empirical density is better described by a $\chi^{2}$ distribution with 11 dof for the $5 \mathrm{yr}$ mission; and with 16 dof for the $10 \mathrm{yr}$ mission (though 15 dof seem to better reproduce the tail at $\Delta \chi^{2} \gtrsim 30$ ).

The cumulative distribution is shown in Fig. 4. The 99\% and 99.73\% quantiles obtained from the empirical distribution are listed in Table 4 . As in the case of $\triangle \mathrm{AIC}$ and $\triangle \mathrm{BIC}$, the quantile increases when going from the $5 \mathrm{yr}$ to the $10 \mathrm{yr}$ mission.

\section{Detection rate}

In this and in the following sections, we consider simulations of one-planet systems. Our samples consist of 4968 and 4706 systems for the $5 \mathrm{yr}$ and $10 \mathrm{yr}$ missions, respectively.

We chose to put our detection threshold at $\triangle \mathrm{BIC}=20$, which is equivalent to $\triangle \mathrm{AIC}=70, \Delta \mathrm{WAIC}=70$, and $\Delta \chi^{2}=80$. This has the effect that, with respect to $\triangle \mathrm{AIC}=20$, the number of false positives is minimised, and the quality of detection is improved 

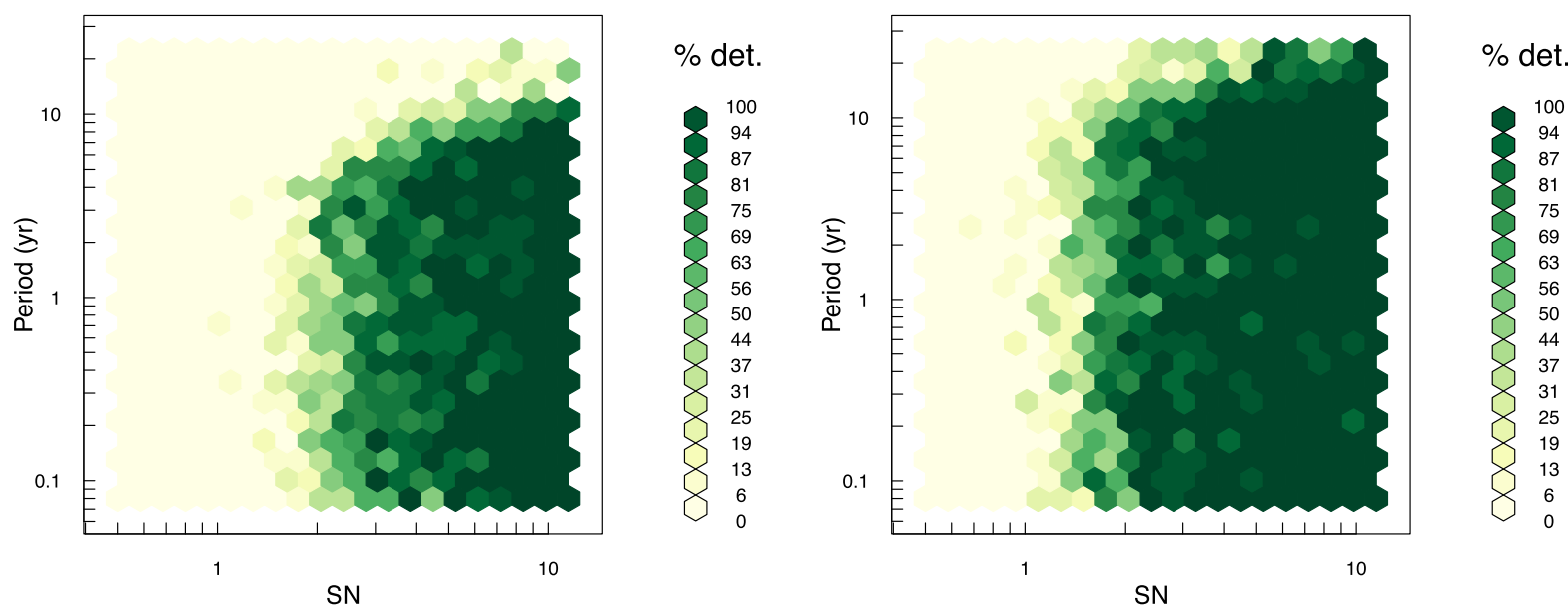

Fig. 5. Detection fractions as a function of $\mathrm{S} / \mathrm{N}$ and period. Left panel: $5 \mathrm{yr}$ mission; right panel: $10 \mathrm{yr}$ mission. The colours show the detection fraction and range from light yellow (low detection fraction) to dark green (high detection fraction). The transition between non-detections and detections is sharper and occurs at lower $\mathrm{S} / \mathrm{N}$ in the $10 \mathrm{yr}$ mission than in the $5 \mathrm{yr}$ mission. Planets with periods longer than the mission length require a stronger $\mathrm{S} / \mathrm{N}$ to be detected than planets with lower periods.

(our tests showed that in systems with very low $\mathrm{S} / \mathrm{N}$ and hence a contrasting AIC versus BIC verdict, the period was incorrectly recovered in approximately one-quarter of the cases).

In Fig. 5 we show the detection fraction as a function of both $\mathrm{S} / \mathrm{N}$ and period. The fraction of detected systems depends mainly on the $\mathrm{S} / \mathrm{N}$. Detecting systems with periods longer than the mission length requires a $\mathrm{S} / \mathrm{N}$ that is higher by an amount that increases with the period.

The $50 \%$ detection rate for systems with period shorter than the mission length is found at $S / N \sim 2.3$ and 1.7 for the $5 \mathrm{yr}$ and $10 \mathrm{yr}$ mission, respectively. Starting the MC with the circular orbit model or at the ideal point makes little difference because the detection rates vary by less than $10 \%$.

The above numbers only consider systems for which the MC achieved convergence within the allowed number of samples, according to the criterion described in Sect. 5.4. We found that the fraction of non-converging chains depends on the starting point: in the ideal starting point case, $99 \%$ of chains converged, while in the case of the circular orbits starting point, the fraction lowers to $\sim 80 \%$. This fraction might increase by using a more refined method to select a starting point (as was done in e.g. Sahlmann et al. 2013).

\section{Parameter recovery}

In this section we consider candidate detections, and look at how well the orbital parameters are recovered. The results depend on how close the initial point for the MC is to the true value of the parameters. To show the impact of the MC starting point, we present the results for the ideal case (starting from true values) for period and eccentricity. We then show, for all orbital parameters, the results obtained by starting the MC at the best-fit points obtained using the circular orbits model.

For every parameter, we calculated the median (the circular median is used for $\Omega, \omega$, and $T_{\mathrm{p}}$; see Pewsey et al. 2013) and an estimate of the associated error: for $\Omega, \omega$, and $T_{\mathrm{p}}$ we used the circular standard deviation ${ }^{8}$; for every other parameter, we

8 Defined as $\hat{\sigma}=\sqrt{-2 \ln \bar{R}}$, where $\bar{R}=\frac{1}{n} \sum_{i=1}^{n} \cos \left(\gamma_{i}-\bar{\gamma}\right)$ is the sample mean resultant length, for a sample of $n$ of angles $\gamma_{i}$ whose mean is $\bar{\gamma}$, and calculated using the sd.circular function in the circular package for the R software (see Pewsey et al. 2013). used the $68.3 \%$ highest posterior density interval (HPDI) from the posterior samples. These quantities are plotted in Figs. 6-10.

\subsection{Period}

Figure 6 (upper panels) shows that the periods are almost always correctly recovered in the ideal case, when the MC are started using the true values of the parameters.

Instead, the use of imperfect starting points produces a small number of non-recovered periods (the systems with $0.1 \lesssim$ $P_{\text {output }} \lesssim 0.3 \mathrm{yr}$ ). Also, a small number of systems are fit with periods that are somewhat shorter or longer than the input value by a factor of $\sim 40-60 \%$ (they are visible in Fig. 6 as the two sequences right above and below the one-to-one line); these systems are also mis-fit as edge-on, high-eccentricity systems (they have incorrect $\cos i_{\text {output }} \sim 0$ and $\left.e_{\text {output }} \sim 1\right)$. The fraction of such systems depends on the mission length but not on the S/N and is, considering only inputs with $0.2 \leq P_{\text {input }} \leq 3.5 \mathrm{yr}, 12 \%$ for the $5 \mathrm{yr}$ mission and $3.2 \%$ for the $10 \mathrm{yr}$ mission. The two sequences can be interpreted as parallel sequences in terms of frequency $f=1 / P$, where the frequency of each sequence is $f_{\text {output }}=f_{\text {input }} \pm 0.3$.

\subsection{Eccentricity}

The eccentricity exhibits quite a large scatter, both in terms of the location of the median $e_{\text {output }}$ and of the size of the HPDI. The scatter is somewhat larger when the MC are started using the circular orbit model.

When the MC are started using the circular orbit model, a fraction of systems are fit with $e_{\text {output }} \sim 1$ with no dependence on $e_{\text {input }}$; this happens in $9.6 \%$ and $6.7 \%$ of the systems with $e_{\text {input }}<$ 0.8 for the $5 \mathrm{yr}$ and $10 \mathrm{yr}$ mission, respectively. Such systems also exhibit incorrect fits in other variables: they have $\omega_{\text {output }} \sim \pm 90^{\circ}$, an incorrect $T_{\mathrm{p}}$, and $\cos i_{\text {output }} \sim 0$ (edge-on); their $P_{\text {output }}$ also run on the parallel sequences to the one-to-one relationships.

\subsection{Size of the orbit}

The angular size of the semi-major axis of the orbit is given by the astrometric signature $v$; its input value $v_{\text {input }}$ can be obtained 

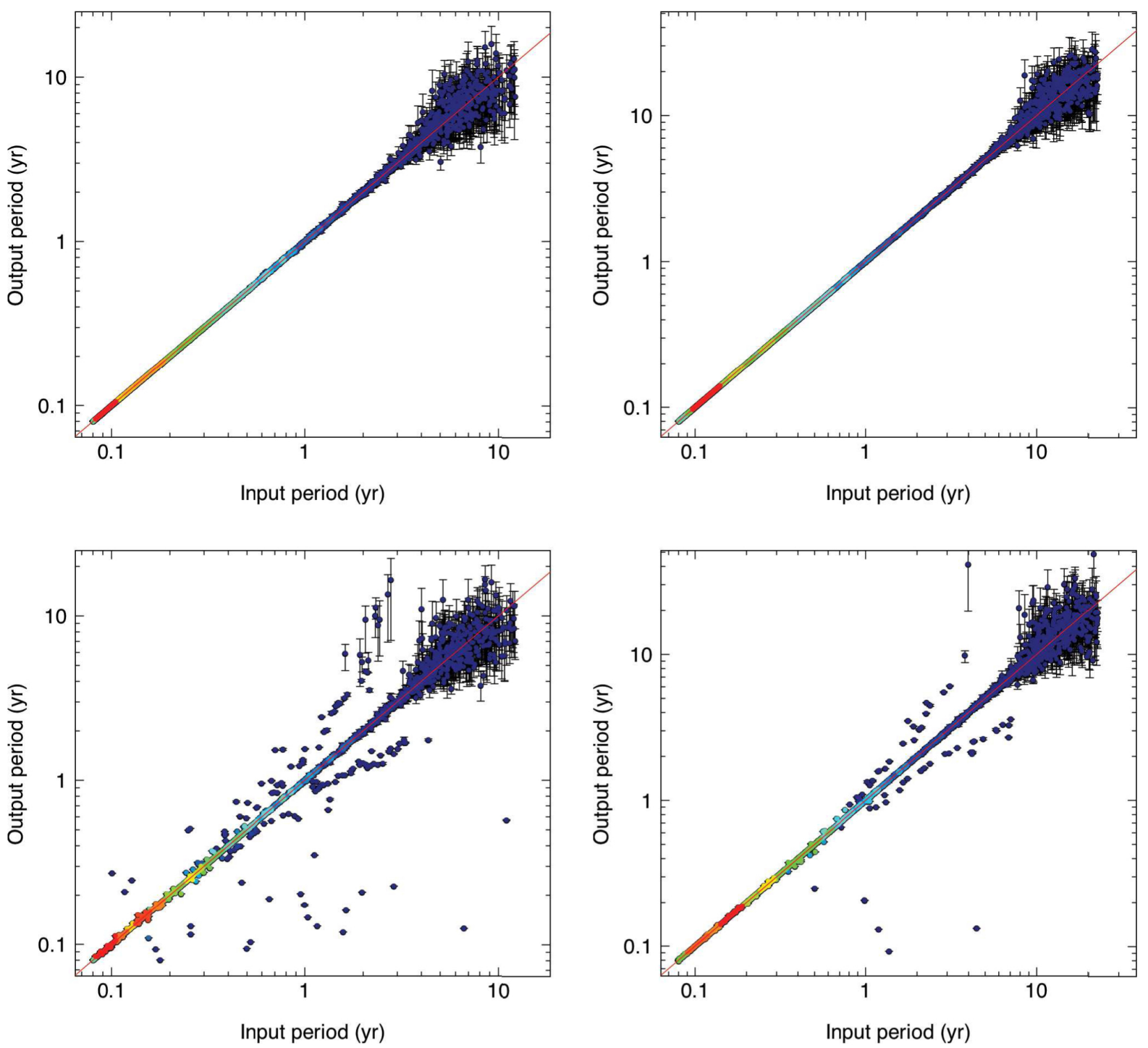

Fig. 6. Simulated vs. recovered period. The left and right panels show the $5 \mathrm{yr}$ and $10 \mathrm{yr}$ missions, respectively. The upper and lower panels differ only for the starting point of the MC: the ideal case (MC started at the true value) in the upper panel, and the circular-orbit model best-fit point in the lower panel. The colours show the density of points where they overlap, going from blue (no overlap) to red (maximum overlap). The error bars show the $68.3 \%$ highest posterior density interval. The red solid lines show the one-to-one relationship. The period is correctly recovered in the majority of cases. The two sequences parallel to the main relationship are populated with systems that are incorrectly fit with edge-on, high-eccentricity orbits.

from the S/N through Eq. (13). In Fig. 8, we show that the oneto-one relationship is well recovered.

Conversely, most of the systems with deviations on the left side (i.e. with $v_{\text {output }}>v_{\text {input }}$ ) are also mis-fit as edge-on, high eccentricity systems $\left(\cos i_{\text {output }} \sim 0\right.$ and $\left.e_{\text {output }} \sim 1\right)$.

\subsection{Inclination}

The inclination is recovered correctly in the majority of cases (Fig. 9); the scatter of the one-to-one relationship is reduced when going from the nominal to the extended mission.

\subsection{Longitude of ascending node}

The longitude of the ascending node $(\Omega)$ is correctly recovered in most cases; see Fig. 10. There does not seem to be any particular structure in the points that deviate most from the one-to-one relationship.

\subsection{Argument of periapsis}

Whether the argument of periapsis $(\omega)$ is correctly recovered depends on the eccentricity. As discussed in Sect. 5.3, when orbits are nearly circular, $\omega$ loses its geometrical meaning and starts to track the zero-point of the orbit as $T_{\mathrm{p}}$. Therefore, for low eccentricity the relationship between $\omega_{\text {input }}$ and $\omega_{\text {output }}$ is lost.

The relationship is recovered for larger eccentricities $e_{\text {input }} \gtrsim$ 0.1 ; the relationship for $\omega$ appears somewhat noisier than the analogous relationships that hold for $\Omega$ and $T_{\mathrm{p}}$.

\subsection{Periapsis transit time}

As already seen in the case of $\omega$, the periapsis transit time $T_{\mathrm{p}}$ is only recovered when orbits are not circular. For systems with $e_{\text {input }}>0.1$, we found that the one-to-one relationship seems well recovered, with no particular structure in the points deviating from it. 

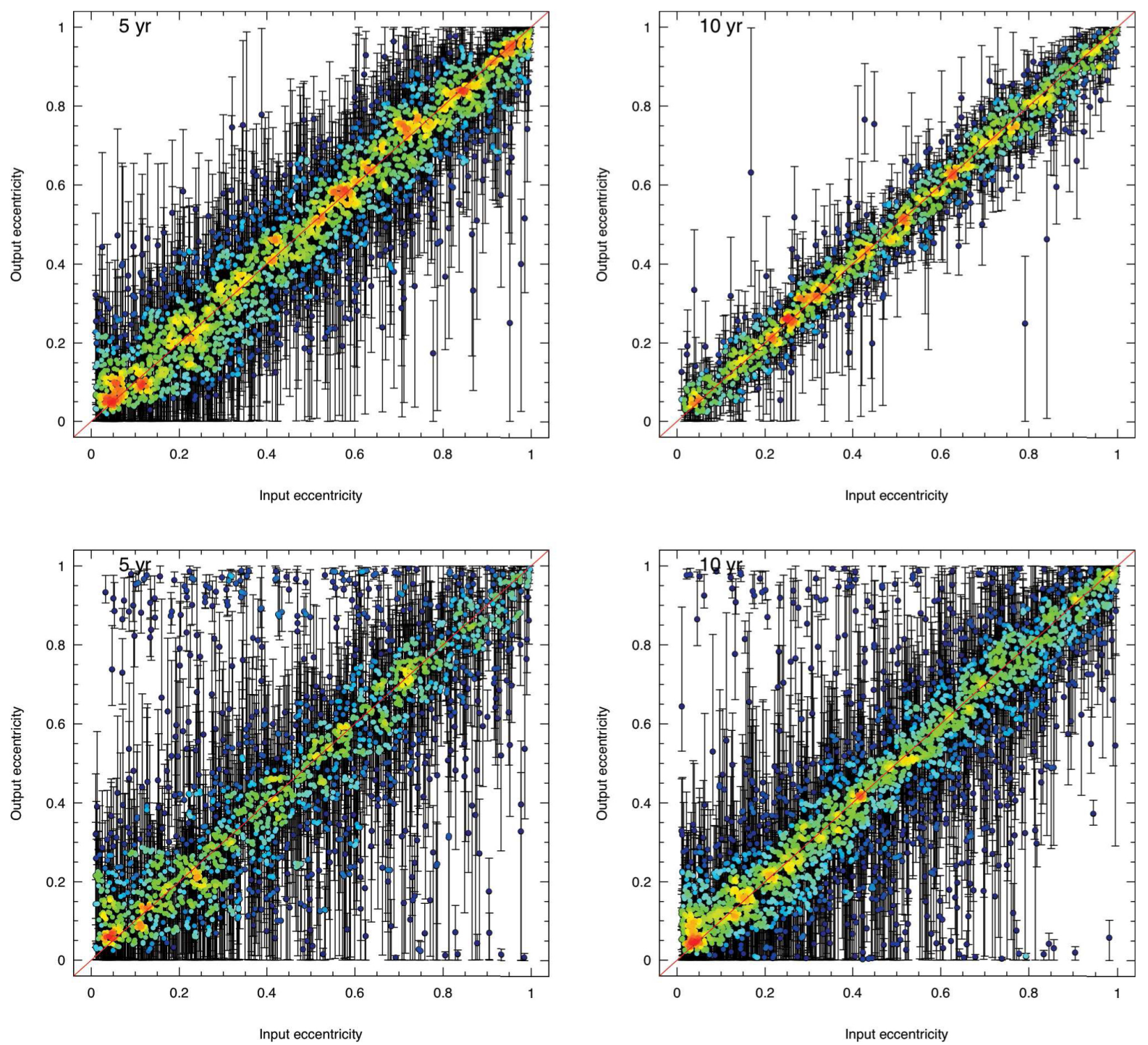

Fig. 7. Simulated vs. recovered eccentricity. Panels, symbols, and colours as in Fig. 6. The eccentricity is recovered in most cases, albeit with large uncertainties. When the MC are started using the circular orbits model (lower panels), some systems with $e_{\text {input }} \lesssim 0.4$ are incorrectly fit with very elliptical orbits $\left(e_{\text {output }} \sim 1\right)$, especially in the $5 \mathrm{yr}$ mission; these systems are the same as those that lie in the parallel sequences of Fig. 6 .

\section{Discussion}

\subsection{Maximum distance of detectable planets}

Previous studies $(\mathrm{C} 08, \mathrm{P} 14)$ had suggested a minimum $S / N=3$ for the detection of planets. This corresponds to a $74 \%$ chance of detecting a planet in the $5 \mathrm{yr}$ mission $(92 \%$ for the $10 \mathrm{yr}$ mission). A $50 \%$ detection chance occurs at $S / N=2.3$ and 1.7 , respectively (Sect. 7).

We can therefore take a second look at Fig. 2, where we present the $\mathrm{S} / \mathrm{N}$ of a few representative classes of planets, as a function of their distance from the solar system. Assuming the $\mathrm{S} / \mathrm{N}$ that allow a $50 \%$ detection chance, the maximum distance up to which a Jupiter-mass planet around a $1 M_{\odot}$ star with a semi-major axis of 1 au could be detected is $13 \mathrm{pc}$ for the $5 \mathrm{yr}$ mission, and $17 \mathrm{pc}$ for the $10 \mathrm{yr}$ mission. Considering a semimajor axis of $3 \mathrm{au}$, the distances would increase to $39 \mathrm{pc}$ and $52 \mathrm{pc}$, respectively. A $10 \mathrm{yr}$ mission also allows the detections of planets with semi-major axes up to 4 au, yielding a maximum distance of $70 \mathrm{pc}$.
For a Neptune-mass planet, the distances are shorter. Even in the favourable case of a 3 au semi-major axis, the distances would be $1.9 \mathrm{pc}$ and $2.6 \mathrm{pc}$, respectively. For the $10 \mathrm{yr}$ mission, a 4 au semi-major axis would yield a distance of $3.5 \mathrm{pc}$. In all cases, there is a better chance to detect planets if one also considers stars with a lower mass than the $1 M_{\odot}$ considered here.

In a real survey, it is possible that some false positives will be included among the detections of planets with low $\mathrm{S} / \mathrm{N}$. The $\Delta \mathrm{BIC}=20$ threshold that we have considered has allowed zero false positives among our no-planet simulations; therefore, considering the number of simulated systems, upper limits to the false positive fraction can be put as $<1.5 \times 10^{-4}$ and $<2.3 \times 10^{-4}$ for the $5 \mathrm{yr}$ and and $10 \mathrm{yr}$ mission, respectively. To put such numbers in the context of a real survey, we consider the number of stars within $100 \mathrm{pc}$ of the Sun. Considering only dwarf stars of the $F, G$, and $K$ spectral types (as done previously by $\mathrm{C} 08$ and P14), the Gaia universe model snapshot (GUMS; Robin et al. 2012) includes $6.3 \times 10^{4}$ stars. Therefore, from the above upper limits to the false positive fraction, one could estimate that $\lesssim 9$ and $\lesssim 14$ false positives could be present in an all-sky survey. 

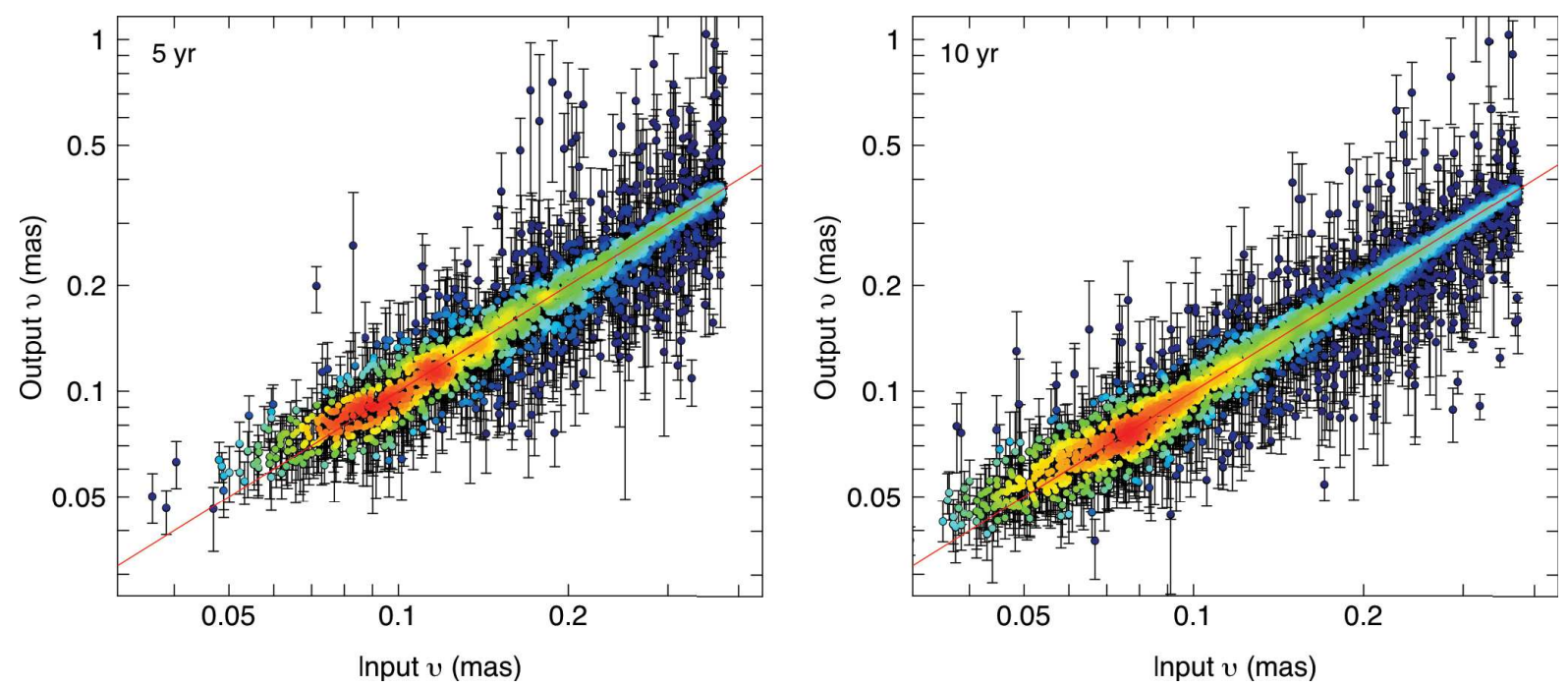

Fig. 8. Simulated vs. recovered angular size of the orbit. Symbols and colours as in Fig. 6. The two panels show only the results when the MC have been started using the circular orbit model. Most of the points with $v_{\text {output }} \ll v_{\text {input }}$ have input periods longer than the mission length, and form the horizontal plume at large $P_{\text {input }}$ in Fig. 6.
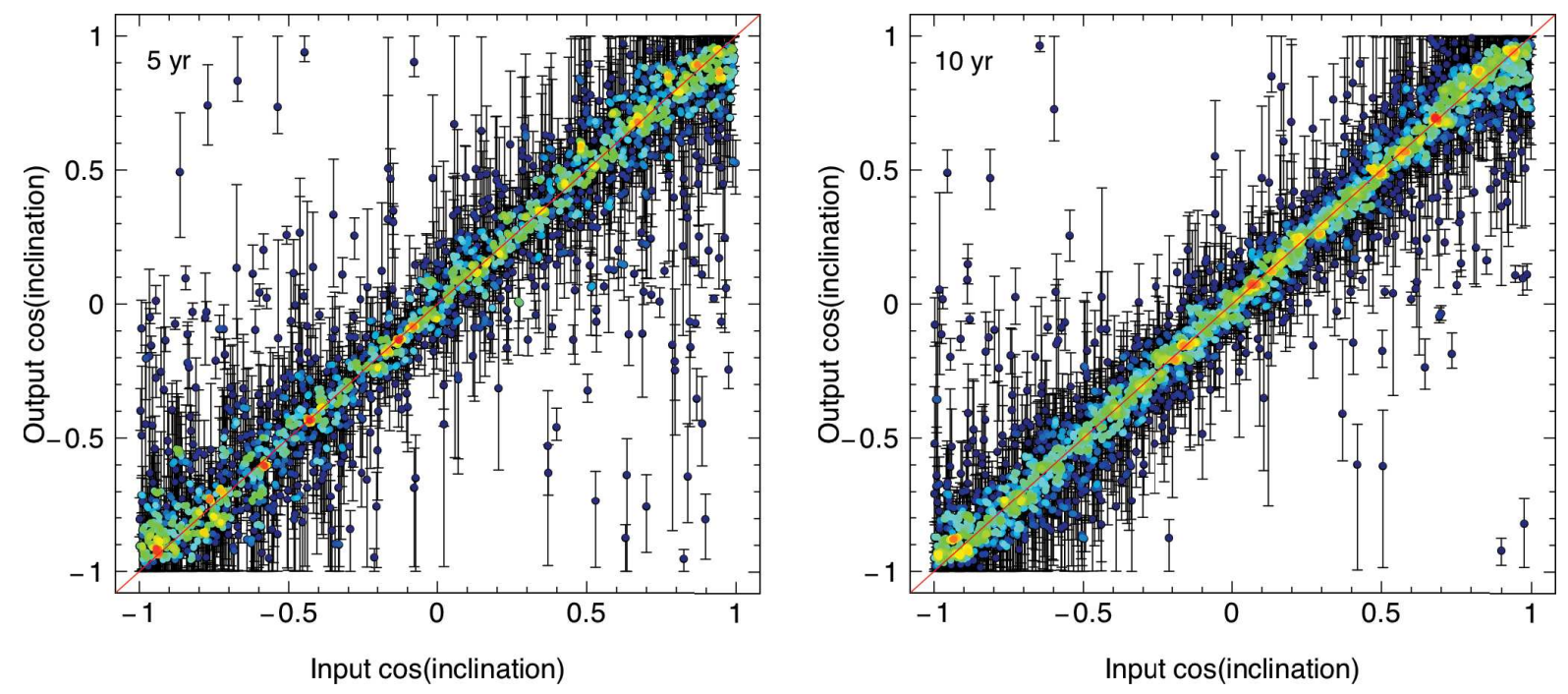

Fig. 9. Simulated vs. recovered inclination. Panels, symbols, and colours as in Fig. 8. The inclination is correctly recovered for most systems. Especially in the $5 \mathrm{yr}$ mission, there is a fraction of systems which are mistaken as edge-on $\left(\cos i_{\text {output }} \sim 0\right)$ which seems to be independent of the input inclination, but which is often associated with high eccentricity $\left(e_{\text {output }} \sim 1\right)$ and with the parallel sequences in the period.

\subsection{Accuracy and precision of fit parameters}

We examine which parameters are recovered better, and with what accuracy and precision they are on average recovered. We define accuracy as the absolute difference between the input and recovered values, and precision as the random error that affects the recovered values (i.e. the length of an error bar, given by the length of the $68.3 \%$ HPDI). For the two scale parameters ( $P$ and $v$, that vary over several orders of magnitude) we consider relative accuracy and precision; for the other parameters, that set the shape of the orbit, we consider absolute accuracy and precision.

In this way we obtain for each parameter a distribution that spans all simulated planetary system; the medians of such distributions can be taken as the characteristic measure of accuracy or precision for each parameter. In Tables 5 and 6 we show the medians of such distributions, along with the $25 \%$ and $75 \%$ quartiles. For example, the period $(P)$ has a relative precision smaller than $\pm 0.41 \%$ in $25 \%$ of the cases in the $5 \mathrm{yr}$ mission.

The accuracy and precision distributions are presented in Fig. 11, where they are shown in the form of a violin plot. In this kind of plot, a kernel density estimate is calculated for each parameter distribution, and plotted twice, to form a symmetric figure with normalised area. At every value of the abscissa, the figure height is proportional to the density of the distribution.

\subsection{Independence from sky position}

We assume in Sect. 4.3 that the star-planet systems are uniformly distributed on the celestial sphere. In principle, this assumption might not necessarily be true. We have found no dependence of either the detection rate or of any aspect of parameter recovery on the sky position; therefore, no position bias should be present in our results. 

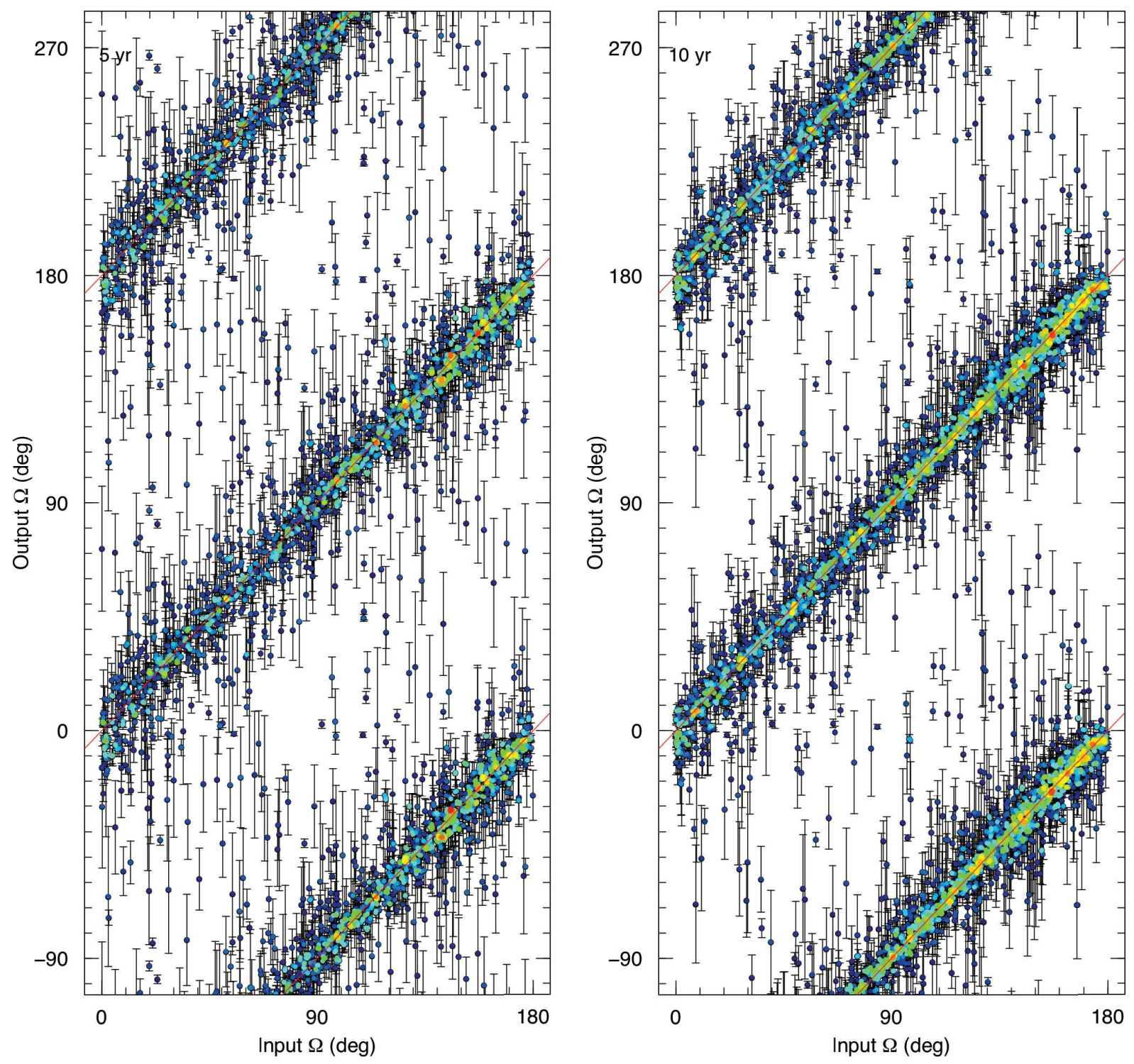

Fig. 10. Simulated vs. recovered longitude of ascending node $(\Omega)$. Panels, symbols, and colours as in Fig. 8. Since $\Omega$ is an angle, we plot the data points twice (at nominal position and at $\pm 180^{\circ}$ ) to better show the structure of the noise.

\section{Conclusions}

We have presented an investigation of the planet detection capabilities of Gaia, using the most up-to-date in-flight properties of the spacecraft. We have tested a simple Bayesian model of astrometric data of planetary orbits that relies on off-theshelf software and standard procedures for inference and model selection.

We have considered two models: one accounting only for astrometric parameters (position, parallax, proper motion), and another accounting for both astrometric and orbital parameters. We investigated model selection using three information criteria (AIC, BIC, WAIC) and the $\Delta \chi^{2}$.

Using simulations of Gaia observations of stars with no planets (one set for a nominal $5 \mathrm{yr}$ mission, and another set for an extended $10 \mathrm{yr}$ mission), we have compared the information criteria against the threshold of $\Delta \mathrm{IC}=20$ suggested for strong evidence in favour of one model over another by Jeffreys' scale for Bayesian evidence. We have found that the use of AIC or WAIC with the above threshold allows false detection fractions that are always $\$ 0.6 \%$, with AIC being somewhat stricter than
Table 5. Distribution of the accuracy with which fit parameters are recovered.

\begin{tabular}{lcccccc}
\hline \hline & \multicolumn{3}{c}{$5 \mathrm{yr}$} & \multicolumn{3}{c}{$10 \mathrm{yr}$} \\
\cline { 2 - 7 } & $25 \%$ & $50 \%$ & $75 \%$ & $25 \%$ & $50 \%$ & $75 \%$ \\
\hline$P$ & $0.14 \%$ & $0.70 \%$ & $6.5 \%$ & $0.058 \%$ & $0.29 \%$ & $1.8 \%$ \\
$v$ & $3.1 \%$ & $8.0 \%$ & $18 \%$ & $2.3 \%$ & $6.0 \%$ & $14 \%$ \\
\hline$e$ & 0.030 & 0.077 & 0.17 & 0.025 & 0.061 & 0.14 \\
$\cos i$ & 0.031 & 0.078 & 0.17 & 0.023 & 0.051 & 0.11 \\
$\Omega$ & $2.7^{\circ}$ & $7.3^{\circ}$ & $25^{\circ}$ & $1.9^{\circ}$ & $5.2^{\circ}$ & $16^{\circ}$ \\
$\omega$ & $8.4^{\circ}$ & $21^{\circ}$ & $45^{\circ}$ & $6.0^{\circ}$ & $18^{\circ}$ & $41^{\circ}$ \\
$T_{\mathrm{p}} / P$ & 0.015 & 0.045 & 0.16 & 0.011 & 0.032 & 0.11 \\
\hline
\end{tabular}

Notes. The first column presents the orbital parameter; the other columns present the distribution of the length of its $68.3 \%$ error bars in the $5 \mathrm{yr}$ mission (Cols. 2-4) and in the $10 \mathrm{yr}$ mission (Cols. 5-7) as the $25 \%$ quartile, median, and $75 \%$ quartile. Relative errors are shown for quantities that vary over several orders of magnitude, namely $P$ and $v$; absolute errors for the remaining parameters. We consider $T_{\mathrm{p}} / P$ (instead of just $T_{\mathrm{p}}$ ) because it is always contained in the $[0,1]$ interval. 

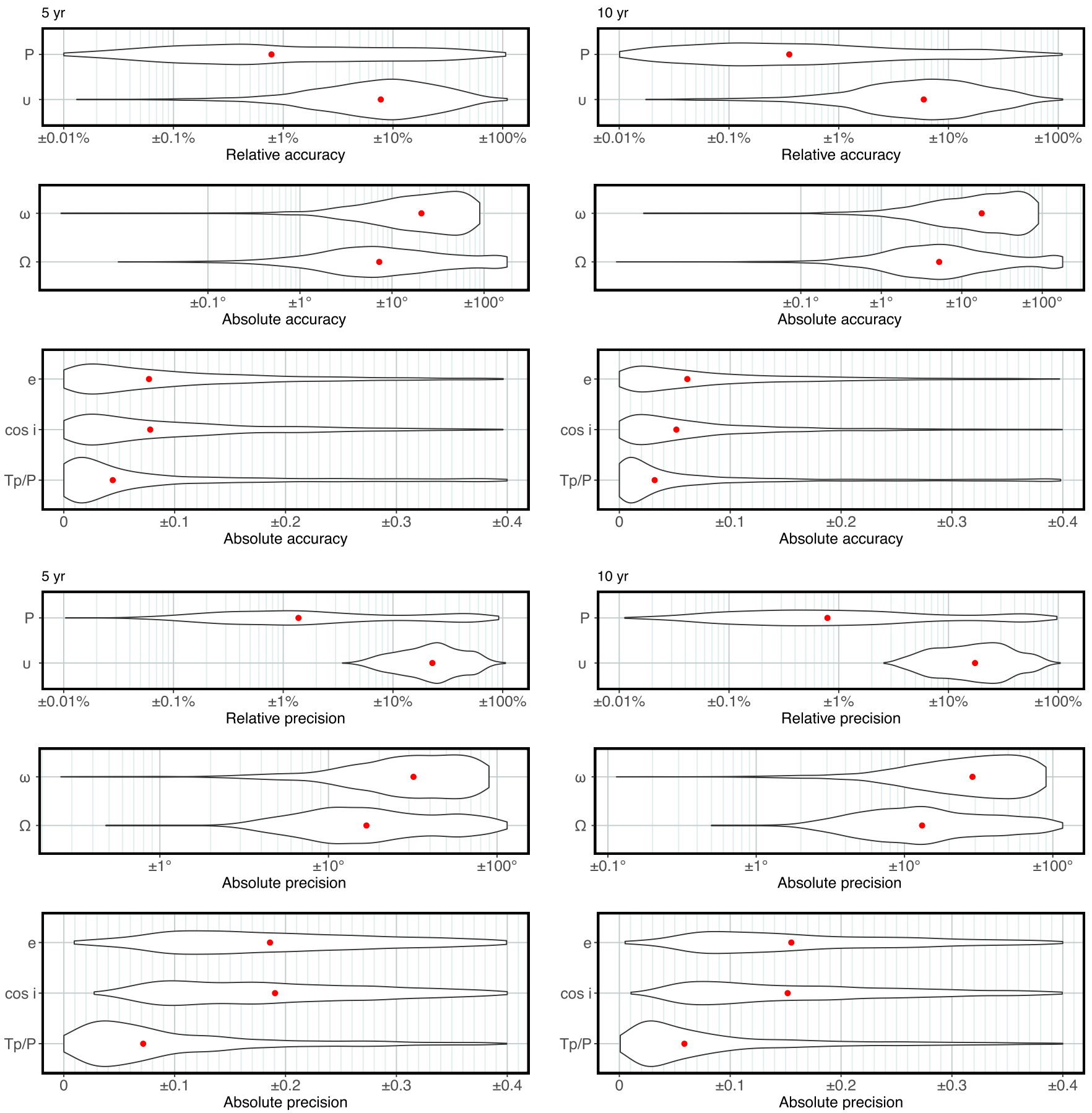

Fig. 11. Accuracy and precision of the fit parameters. Left panels: $5 \mathrm{yr}$ mission; right panels: $10 \mathrm{yr}$ mission. For each orbital parameter, the accuracy is the absolute difference between the input value and the median of the output posterior distribution. The precision is the length of the $68.3 \%$ highest posterior density interval (HPDI). The figures depicted by the black curves show the distribution of either accuracy or precision; they have normalised areas, so that at every value of the abscissa, their height is proportional to the density of the distribution. The red dots mark the median of each distribution. Relative quantities are shown for quantities that vary over several orders of magnitude, namely $P$ and $v$; absolute quantities for the remaining parameters. We consider $T_{\mathrm{p}} / P$ (instead of just $T_{\mathrm{p}}$ ) because it is always contained in the $[0,1]$ interval; thus, $T_{\mathrm{p}}$ has a median precision of $5 \% P$ in the $5 \mathrm{yr}$ mission.

WAIC. The BIC criterion, that puts a stronger penalty on the more complex model, allowed no false positive out of 6644 cases ( $5 \mathrm{yr})$ and 4402 cases $(10 \mathrm{yr})$. The $\Delta \chi^{2}$ can also be used, but the non-linearity of the model prevents the use of a reference $\chi^{2}$ distribution to obtain a $p$-value; therefore the choice of a $\Delta \chi^{2}$ threshold needs an empirical calibration.

We have investigated parameter recovery using simulations of stars with one planet, whose $\mathrm{S} / \mathrm{N}$ and periods were assigned according to a logarithmically spaced grid, and whose eccentricities were assigned on a linear grid. All other parameters are assigned randomly. Realistic scanning laws are calculated according to a randomly assigned position on the sky.

We have applied our detection method to 4968 and 4706 simulations of stars with one planet over a mission of $5 \mathrm{yr}$ and $10 \mathrm{yr}$, respectively. We have chosen a detection threshold of $\triangle \mathrm{BIC}=20$ (i.e. with $\mathrm{BIC}$ at the strong evidence point 
Table 6. Distribution of the precision with which the fit parameters are recovered.

\begin{tabular}{lrrrrrr}
\hline \hline & \multicolumn{3}{c}{$5 \mathrm{yr}$} & \multicolumn{3}{c}{$10 \mathrm{yr}$} \\
\cline { 2 - 7 } & $25 \%$ & $50 \%$ & $75 \%$ & $25 \%$ & $50 \%$ & $75 \%$ \\
\hline$P$ & $0.40 \%$ & $1.4 \%$ & $7.3 \%$ & $0.19 \%$ & $0.79 \%$ & $3.8 \%$ \\
$v$ & $14 \%$ & $23 \%$ & $35 \%$ & $9.0 \%$ & $17 \%$ & $30 \%$ \\
\hline$e$ & 0.12 & 0.19 & 0.28 & 0.094 & 0.16 & 0.26 \\
$\cos i$ & 0.12 & 0.19 & 0.29 & 0.087 & 0.15 & 0.25 \\
$\Omega$ & $9.2^{\circ}$ & $17^{\circ}$ & $37^{\circ}$ & $6.7^{\circ}$ & $13^{\circ}$ & $30^{\circ}$ \\
$\omega$ & $18^{\circ}$ & $32^{\circ}$ & $54^{\circ}$ & $15^{\circ}$ & $29^{\circ}$ & $50^{\circ}$ \\
$T_{\mathrm{p}} / P$ & 0.036 & 0.072 & 0.14 & 0.029 & 0.059 & 0.13 \\
\hline
\end{tabular}

Notes. Columns as in Table 5.

according to Jeffreys' scale) which is equivalent to $\triangle \mathrm{AIC}=70$, $\Delta \mathrm{BIC}=20, \Delta \chi^{2}=80$. This not only minimises the number of false positives, but has also guaranteed that the detected systems show a good quality of fit.

We have confirmed the finding of previous studies that the $\mathrm{S} / \mathrm{N}$ is the main parameter that determines whether a planet can be detected. The $50 \%$ detection threshold occurs at $S / N=2.3$ and 1.7 for the $5 \mathrm{yr}$ and $10 \mathrm{yr}$ mission, respectively. Therefore, some approximate maximum distances to which planets around a $1 M_{\odot}$ star can be detected with a $50 \%$ chance are 39 pc (a Jupiter-mass planet orbiting at 3 au; $5 \mathrm{yr}$ mission), 70 pc (Jupitermass, 4 au, $10 \mathrm{yr}$ ), $1.9 \mathrm{pc}$ (Neptune-mass, $3 \mathrm{au}, 5 \mathrm{yr}$ ), and $3.5 \mathrm{pc}$ (Neptune-mass, 4 au, $10 \mathrm{yr}$ ).

The orbital parameters of planets with periods up to the mission length can be recovered with different degrees of accuracy and precision. We define accuracy as the difference between the input value and the median of the output posterior distribution, and precision as the length of the $68.3 \%$ HPDI. Considering the period, in the case of a $5 \mathrm{yr}$ mission we find a median relative accuracy of $0.70 \%$ and a median relative precision of $1.4 \%$. For the angular size of the semi-major axis of the orbit, we find $7.9 \%$ and $23 \%$, respectively. For the eccentricity, we find 0.077 and 0.19 , respectively.

A follow-up of this work should deal with the detectability of multiple planets in the same systems, and of very long-period planets, and should consider data from both Gaia and a future astrometric mission.

Acknowledgements. We thank an anonymous referee whose comments have greatly contributed to improve this paper. We thank Martin Gustavsson for providing an initial version of our simulation code, and Alessandro Sozzetti for useful discussions. We gratefully acknowledge support from the Swedish National Space Board (SNSB Dnr 183/14 and SNSB Dnr 74/14) and the Royal Physiographic Society in Lund.

\section{References}

Akaike, H. 1973, in 2nd International Symposium on Information Theory, Academiai Kiado, reprinted in Selected Papers of Hirotugu Akaike (Berlin, Heidelberg: Springer, 1998), 199

Andrae, R., Schulze-Hartung, T., \& Melchior, P. 2010 ArXiv e-prints [arXiv: 1012.3754]

Bernstein, H.-H., \& Bastian, U. 1995, in Proc. of the RGO - Future Possibilities for Astrometry in Space, eds. M. A. C. Perryman, \& F. van Leeuwen, ESA SP, 379, 55
Binnendijk, L. 1960, Properties of Double Stars: A Survey of Parallaxes and Orbits (Philadelphia: Pennsylvania University Press)

Boss, A. P., Butler, R. P., Hubbard, W. B., et al. 2007, IAU Trans., 26A, 183

Burnham, K. P., \& Anderson, D. R. B. 2002, Model Selection and Multimodel Inference: A Practical Information-theoretic Approach (New York: Springer) Carpenter, B., Gelman, A., Hoffman, M., et al. 2016, J. Stat. Softw., 76, 20

Casertano, S., Lattanzi, M. G., \& Perryman, M. A. C. 1995, in Future Possibilities for astrometry in Space, eds. M. A. C. Perryman, \& F. van Leeuwen, ESA SP, 47

Casertano, S., Lattanzi, M. G., Sozzetti, A., et al. 2008, A\&A, 482, 699

de Bruijne, J., Siddiqui, H., Lammers, U., et al. 2010, in Relativity in Fundamental Astronomy: Dynamics, Reference Frames, and Data Analysis, eds. S. A. Klioner, P. K. Seidelmann, \& M. H. Soffel, IAU Symp., 261, 331

de Bruijne, J. H. J. 2012, Ap\&SS, 341, 31

de Bruijne, J. H. J., Rygl, K. L. J., \& Antoja, T. 2014, in EAS Pub. Ser., 67, 23

Efron, B., \& Gous, A. 2001, in Model Selection, ed. P. Lahiri, IMS Lecture Notes Monograph Series, 38, 208

ESA 1997, The HIPPARCOS and TYCHO catalogues. Astrometric and Photometric Star Catalogues Derived from the ESA HIPPARCOS Space Astrometry Mission, ESA SP, 1200

Gaia Collaboration (Brown, A. G. A., et al.) 2016a, A\&A, 595, A2

Gaia Collaboration (Prusti, T., et al.) 2016b, A\&A, 595, A1

Gatewood, G., Han, I., \& Black, D. C. 2001, ApJ, 548, L61

Gelman, A., \& Rubin, D. B. 1992, Stat. Sci., 7, 457

Gelman, A., Carlin, J., Stern, H., et al. 2013, Bayesian Data Analysis, 3rd edn., (Boca Raton, FL: CRC Press)

Geyer, C. 2011, in Handbook of Markov Chain Monte Carlo Handbooks of Modern Statistical Methods, eds. S. Brooks, A. Gelman, G. L. Jones, \& X.-L. Meng (London: Chapman and Hall/CRC), 3

Gregory, P. 2005, Bayesian Logical Data Analysis for the Physical Sciences: A Comparative Approach with Mathematica ${ }^{\circledR}$ Support (Cambridge: Cambridge University Press)

Han, I., Black, D. C., \& Gatewood, G. 2001, ApJ, 548, L57

Holl, B., Lindegren, L., \& Hobbs, D. 2012, A\&A, 543, A15

Jacob, W. S. 1855, MNRAS, 15, 228

Jammalamadaka, S., \& SenGupta, A. 2001, in Series on Multivariate Analysis, ed. M. M. Rao (Singapore: World Scientific), 5

Liddle, A. R. 2007, MNRAS, 377, L74

Lindegren, L., Lammers, U., Hobbs, D., et al. 2012, A\&A, 538, A78

Lindegren, L., Lammers, U., Bastian, U., et al. 2016, A\&A, 595, A4

Lunn, D. J., Thomas, A., Best, N., \& Spiegelhalter, D. 2000, Stat. Comput., 10, 325

Markley, F. L. 1995, Celest. Mech. Dyn. Astron., 63, 101

Mazeh, T., Zucker, S., Dalla Torre, A., \& van Leeuwen, F. 1999, ApJ, 522, L149

Michalik, D., Lindegren, L., Hobbs, D., \& Lammers, U. 2014, A\&A, 571, A85

Mikkola, S. 1987, Celestial Mechanics, 40, 329

Perryman, M. A. C. 2011, The Exoplanet Handbook (Cambridge, NY: Cambridge University Press)

Perryman, M., Hartman, J., Bakos, G. Á., \& Lindegren, L. 2014, ApJ, 797, 14

Pewsey, A., Neuhäuser, M., \& Ruxton, G. 2013, Circular Statistics in R, EBLSchweitzer (Oxford: Oxford University Press)

Plummer, M. 2003, in Proceedings of the 3rd International Workshop on Distributed Statistical Computing in Vienna. vol. 124, eds. K., Hornik, F., Leisch \& A., Zeileis, 125

Pourbaix, D. 2001, A\&A, 369, L22

Quirrenbach, A. 2010, in Exoplanets, ed. S. Seager (Tucson: The University of Arizona Press), 157

Ranalli, P., Koulouridis, E., Georgantopoulos, I., et al. 2016, A\&A, 590, A80

Robin, A. C., Luri, X., Reylé, C., et al. 2012, A\&A, 543, A100

Sahlmann, J., Lazorenko, P. F., Ségransan, D., et al. 2013, A\&A, 556, A133

Schwarz, G. 1978, Ann. Statist., 6, 461

Taff, L. G. 1985, Celestial Mechanics: A Computational Guide for the Practitioner (New York: Wiley-Interscience)

Thiele, T. N. 1883, Astron. Nachr., 104, 245

Trotta, R. 2008, Contemporary Phys., 49, 71

van den Bos, W. H. 1926, CIUO, 68, 352

van den Bos, W. H. 1932, CIUO, 86, 261

Watanabe, S. 2010, J. Mach. Learn. Res., 11, 3571

Watanabe, S. 2013, J. Mach. Learn. Res., 14, 867

Zucker, S., \& Mazeh, T. 2000, ApJ, 531, L67 